\title{
A Family of Trigonometrically Fitted Enright Second Derivative Methods for Stiff and Oscillatory Initial Value Problems
}

\author{
F. F. Ngwane ${ }^{1}$ and S. N. Jator ${ }^{2}$ \\ ${ }^{1}$ Department of Mathematics, USC Salkehatchie, Walterboro, SC 29488, USA \\ ${ }^{2}$ Department of Mathematics and Statistics, Austin Peay State University, Clarksville, TN 37044, USA \\ Correspondence should be addressed to F. F. Ngwane; fifonge@yahoo.com
}

Received 23 January 2015; Accepted 23 April 2015

Academic Editor: Mehmet Sezer

Copyright (c) 2015 F. F. Ngwane and S. N. Jator. This is an open access article distributed under the Creative Commons Attribution License, which permits unrestricted use, distribution, and reproduction in any medium, provided the original work is properly cited.

A family of Enright's second derivative formulas with trigonometric basis functions is derived using multistep collocation method. The continuous schemes obtained are used to generate complementary methods. The stability properties of the methods are discussed. The methods which can be applied in predictor-corrector form are implemented in block form as simultaneous numerical integrators over nonoverlapping intervals. Numerical results obtained using the proposed block form reveal that the new methods are efficient and highly competitive with existing methods in the literature.

\section{Introduction}

Many real life processes in areas such as chemical kinetics, biological sciences, circuit theory, economics, and reactions in physical systems can be transformed into systems of ordinary differential equations (ODE) which are generally formulated as initial value problems (IVPs). Some classes of IVPs are stiff and/or highly oscillatory as described by the following model problem:

$$
\begin{array}{r}
y^{\prime}=A y, \\
y(a)=y_{0}, \\
x \in[a, b],
\end{array}
$$

where $y(x) \in \mathbb{R}^{m}$ and $A$ is $m \times m$ real matrix with at least one eigenvalue with a very negative real part and/or very large imaginary part, respectively (see Fatunla [1]). Many conventional methods cannot solve these types of problems effectively.

Stiff systems have been solved by several authors including Lambert [2, 3], Gear [4, 5], Hairer [6], and Hairer and Wanner [7]. Different methods including the Backward Differentiation Formula (BDF) have been used to solve stiff systems. Second derivative methods with polynomial basis functions were proposed to overcome the Dahlquist [8] barrier theorem whereby the conventional linear multistep method was modified by incorporating the second derivative term in the derivation process in order to increase the order of the method, while preserving good stability properties (see Gear [9], Gragg and Stetter [10], and Butcher [11]).

Many classical numerical methods including RungeKutta methods, higher derivative multistep schemes, and block methods have been constructed for solving oscillatory initial value problems (see Butcher [11, 12], Brugnano and Trigiante [13, 14], Ozawa [15], Nguyen et al. [16], Berghe and van Daele [17], Vigo-Aguiar and Ramos [18], and Calvo et al. [19]). Many methods for solving oscillatory IVPs require knowledge of the system under consideration in advance.

Obrechkoff [20] proposed a general multiderivative method for solving systems of ordinary differential equations. Special cases of Obrechkoff method have been developed by many others including Cash [21] and Enright [22]. The methods by Enright [22] have order $p=k+2$ for a $k$ step method.

In this paper, we propose a numerical integration formula which more effectively copes with stiff and/or oscillatory 
IVPs. We will construct a continuous form of the second derivative multistep method (CSDMM) using a multistep collocation technique such that Enright's second derivative methods (ESDM) will be recovered from the derived continuous methods. The aim of this paper is to derive a family of Enright's second derivative formulas with trigonometric basis functions using multistep collocation method. Many methods for solving IVPs are implemented in a step-by-step fashion in which, on the partition $\pi_{N}$, an approximation is obtained at $x_{n+1}$ only after an approximation at $x_{n}$ has been computed, where $\pi_{N}: a=x_{0}<x_{1}<\cdots<x_{n}<x_{n+1}<\cdots<$ $x_{N}=b, x_{n+1}=x_{n}+h, n=1, \ldots, N, h=(b-a) / N, h$ is the step size, $N$ is a positive integer, and $n$ is the grid index. We implement ESDM in block form.

In Section 2, we present a derivation of the family of Enright methods. Error analysis and stability are discussed in Section 3. The implementation of the ESDM and numerical examples to show the accuracy and efficiency of the ESDM are given in Section 4. Finally, we conclude in Section 5.

\section{Derivation of the Family of Methods}

We consider the first-order differential equation

$$
\begin{gathered}
y^{\prime}=f(x, y), \\
y(a)=y_{0}, \\
x \in[a, b],
\end{gathered}
$$

where $f$ is assumed to satisfy the conditions to guarantee the existence of a unique solution of the initial-value problem.

2.1. CSDMM. In what follows, we state the CSDMM which has the ability to produce the ESDM:

$$
\begin{aligned}
U(x)= & \alpha_{n+k-1}(x) y_{n+k-1}+h \sum_{j=0}^{k} \beta_{j}(x) f_{n+j} \\
& +h^{2} \gamma_{n+k}(x) g_{n+k},
\end{aligned}
$$

where $\alpha_{n+k-1}(x), \beta_{j}(x)$, and $\gamma_{n+k}(x)$ are continuous coefficients. We assume that $y_{n+j}=U\left(x_{n+j}\right)$ is the numerical approximation to the analytical solution $y\left(x_{n+j}\right), y_{n+j}^{\prime}=$ $U^{\prime}\left(x_{n+j}\right)$ is the numerical approximation to the analytical solution $y^{\prime}\left(x_{n+j}\right), f_{n+j}=U^{\prime}\left(x_{n+j}\right)$ is an approximation to $y^{\prime}\left(x_{n+j}\right)$, and $g_{n+j}=U^{\prime \prime}\left(x_{n+j}\right)$ is an approximation to $y^{\prime \prime}\left(x_{n+j}\right)$, where $f_{n+j}=f\left(x_{n+j}, y_{n+j}\right), g_{n+j}=$ $\left.(d f(x, y(x)) / d x)\right|_{y_{n+j}} ^{x_{n+j}}, j=0,1,2, \ldots, k$.
We now define the following vectors and matrix used in the following theorem:

$$
\begin{aligned}
& V=\left(y_{n+k-1}, f_{n}, f_{n+1}, f_{n+2}, \ldots, f_{n+k}, g_{n+k}\right)^{T}, \\
& P(x)=\left(P_{1}(x), P_{2}(x), \ldots, P_{k+3}(x)\right)^{T}, \\
& W_{(k+3, k+3)} \\
& =\left(\begin{array}{cccc}
P_{1}\left(x_{n+k-1}\right) & P_{2}\left(x_{n+k-1}\right) & \cdots & P_{k+3}\left(x_{n+k-1}\right) \\
P_{1}^{\prime}\left(x_{n}\right) & P_{2}^{\prime}\left(x_{n}\right) & \cdots & P_{k+3}^{\prime}\left(x_{n}\right) \\
P_{1}^{\prime}\left(x_{n+1}\right) & P_{2}^{\prime}\left(x_{n+1}\right) & \cdots & P_{k+3}^{\prime}\left(x_{n+1}\right) \\
P_{1}^{\prime}\left(x_{n+2}\right) & P_{2}^{\prime}\left(x_{n+2}\right) & \cdots & P_{k+3}^{\prime}\left(x_{n+2}\right) \\
\vdots & \vdots & \ddots & \vdots \\
P_{1}^{\prime}\left(x_{n+k}\right) & P_{2}^{\prime}\left(x_{n+k}\right) & \cdots & P_{k+3}^{\prime}\left(x_{n+k}\right) \\
P_{1}^{\prime \prime}\left(x_{n+k}\right) & P_{2}^{\prime \prime}\left(x_{n+k}\right) & \cdots & P_{k+3}^{\prime \prime}\left(x_{n+k}\right)
\end{array}\right),
\end{aligned}
$$

where $P_{i}(x)=x^{i-1}, i=1,2, \ldots, k+1, P_{k+2}(x)=\sin w x$, and $P_{k+3}(x)=\cos w x$.

Remark 1. In the derivation of the ESDM, the bases $P(x) \equiv$ $P_{i}(x)^{T}$ with $P_{i}(x)=x^{i-1}, i=1,2, \ldots, k+1, P_{k+2}(x)=\sin (w x)$, and $P_{k+3}(x)=\cos (w x)$ are chosen because they are simple to analyze. Other possible bases (see Nguyen et al. [16] and Nguyen et al. [23]) include the following:

(1) $\{\sin (w x), \cos (w x), x \sin (w x), x \cos (w x), \ldots$ $\left.x^{n} \sin (w x), x^{n} \cos (w x)\right\}$

(2) $\{\sin x, \cos x, \ldots, \sin (n w x), \cos (n w x)\}$;

(3) $\left\{\sin \left(w_{1} x\right), \cos \left(w_{1} x\right), \ldots, \sin \left(w_{n} x\right), \cos \left(w_{n} x\right)\right\}$;

(4) $\left\{x, \ldots, w x^{n}\right\} \cup\{\sin (w x), \cos (w x), \ldots, \sin (m w x)$, $\cos (m w x)\}$

(5) $\left\{x, \ldots, x^{n}, \exp ( \pm w x), x \exp ( \pm w x), \ldots, x^{m} \exp ( \pm w x)\right\}$;

(6) $\left\{x, \ldots, w x^{n-1}, w x^{n}\right\}$.

Theorem 2. Let $U(x)$ satisfy $U\left(x_{n+j}\right)=y_{n+j}, U^{\prime}\left(x_{n+j}\right)=f_{n+j}$, and $U^{\prime \prime}\left(x_{n+j}\right)=g_{n+j}$ and let $W$ be invertible; then method (3) is equivalent to

$$
U(x)=V^{T}\left(W^{-1}\right)^{T} P(x) .
$$

The proof of the above theorem can be found in Jator et al. [24].

Through interpolation of $U(x)$ at the point $x_{n+k-1}$, collocation of $U^{\prime}(x)$ at the points $x_{n+j}, j=0,1,2, \ldots, k$, and collocation of $U^{\prime \prime}(x)$ at the point $x_{n+k}$, we get the system

$$
\begin{aligned}
U\left(x_{n+k-1}\right) & =y_{n+k-1}, \\
U^{\prime}\left(x_{n+j}\right) & =f_{n+j} \quad j=0,1,2, \ldots, k, \\
U^{\prime \prime}\left(x_{n+k}\right) & =g_{n+k} .
\end{aligned}
$$


To solve this system we require that method (3) be defined by the assumed basis functions

$$
\begin{aligned}
\alpha_{n+k-1} & =\sum_{i=0}^{k+3} \alpha_{i} P_{i}(x) \\
h \beta_{j}(x) & =\sum_{i=0}^{k+3} h \beta_{i, j} P_{i}(x), \quad j=0,1, \ldots, k \\
h^{2} \gamma_{n+k} & =\sum_{i=0}^{k+3} h^{2} \gamma_{i} P_{i}(x)
\end{aligned}
$$

where the constants $\alpha_{i}, \beta_{i, j}$, and $\gamma_{i}$ are to be determined.

2.2. ESDM. The general second derivative formula for solving (2) using the $k$-step second derivative linear multistep method is of the form

$$
\sum_{j=0}^{k} \alpha_{j} y_{n+j}=h \sum_{j=0}^{k} \beta_{j} f_{n+j}+h^{2} \sum_{j=0}^{k} \gamma_{j} g_{n+j}
$$

where $y_{n+j} \approx y\left(x_{n}+j h\right), f_{n+j}=f\left(x_{n}+j h, y\left(x_{n}+j h\right)\right)$ and $f_{j}=$ $f\left(x_{j}, y_{j}\right), g_{n+j}=\left.(d f(x, y(x)) / d x)\right|_{y_{n+j}} ^{x_{n+j}} ; x_{n}$ is a discrete point at node $n$; and $\alpha_{j}, \beta_{j}$, and $\gamma_{j}$ are parameters to be determined. It is worth noting that Enright's method is a special case of (7). We solve (6) to get the coefficients $\alpha_{i}, \beta_{i, j}$, and $\gamma_{i}$ in (7) which are then used to obtain the continuous multistep method of Enright in the form

$$
U(x)=y_{n+k-1}+h \sum_{j=0}^{k} \beta_{j}(x) f_{n+j}+h^{2} \gamma_{n+k}(x) g_{n+k} \text {. }
$$

Evaluating (9) at $x=x_{n+k}$ and setting $y_{n+k}=U\left(x_{n}+k h\right)$ yield the following Enright's second derivative multistep method:

$$
y_{n+k}=y_{n+k-1}+h \sum_{j=0}^{k} \beta_{j} f_{n+j}+h^{2} \gamma_{n+k} g_{n+k},
$$

whereas the $(k-1)$ complementary methods

$$
y_{n+i}=y_{n+k-1}+h \sum_{j=0}^{k} \widehat{\beta}_{j, i} f_{n+j}+h^{2} \widehat{\gamma}_{n+k, i} g_{n+k}
$$

are obtained by evaluating (9) at $x=x_{n+i}, i=0,1,2, \ldots, k-2$, with $k \geq 2$.

We note that, in order to avoid the cancellations which might occur when $h$ is small, the use of the power series expansions of $\beta_{j}, \gamma_{n+k}, \widehat{\beta}_{j, i}$, and $\widehat{\gamma}_{n+k, i}$ is preferable (see Simos [25]).
Case $k=1$. This case has only the main method given by (10) with the coefficients defined by

$$
\begin{aligned}
\beta_{0}= & -\frac{\csc (u / 2)^{2}(-u+\sin u)}{2 u} \\
= & \frac{1}{3}+\frac{u^{2}}{90}+\frac{u^{4}}{2520}+\frac{u^{6}}{75600}+\frac{u^{8}}{2395008} \\
& +\frac{691 u^{10}}{54486432000}+O\left(h^{11}\right) \\
\beta_{1}= & -\frac{\csc (u / 2)^{2}(u \cos u-\sin u)}{2 u} \\
= & \frac{2}{3}-\frac{u^{2}}{90}-\frac{u^{4}}{2520}-\frac{u^{6}}{75600}-\frac{u^{8}}{2395008} \\
& -\frac{691 u^{10}}{54486432000}+O\left(h^{11}\right), \\
\gamma_{n+1}= & -2+u \cot (u / 2) \\
u^{2} & -\frac{1}{6}-\frac{u^{2}}{360}-\frac{u^{4}}{15120}-\frac{u^{6}}{604800}-\frac{u^{8}}{23950080} \\
& -O\left(h^{11}\right) .
\end{aligned}
$$

Case $k=2$. The coefficients of the main method (10) and the complimentary method (11) are, respectively, defined by

$$
\begin{aligned}
\beta_{0}= & \frac{h \csc (u / 2)^{2}(u \cos (u / 2)-2 \sin (u / 2))^{2}}{4 u(u \cos u-\sin u)} \\
= & -\frac{1}{48}-\frac{u^{2}}{360}-\frac{13 u^{4}}{57600}-\frac{89 u^{6}}{6048000}-\frac{143203 u^{8}}{167650560000} \\
& -\frac{126473 u^{10}}{2724321600000}+O\left(h^{11}\right), \\
\beta_{1} & \\
= & \frac{\csc (u / 2)^{2}\left(-2-3 u^{2}+\left(2-u^{2}\right) \cos 2 u+4 u \sin u+2 u \sin 2 u\right)}{8 u(u \cos u-\sin u)} \\
= & \frac{5}{12}+\frac{u^{2}}{720}+\frac{13 u^{4}}{50400}+\frac{121 u^{6}}{6048000}+\frac{52133 u^{8}}{41912640000} \\
& +\frac{761473 u^{10}}{10897286400000}+O\left(h^{11}\right), \\
\beta_{2} & \\
= & -\frac{h \csc (u / 2)^{2}\left(2-\left(4+3 u^{2}\right) \cos u+\left(2+u^{2}\right) \cos 2 u+4 u \sin u\right)}{8 u(u \cos u-\sin u)} \\
= & \frac{29}{48}+\frac{u^{2}}{720}-\frac{13 u^{4}}{403200}-\frac{u^{6}}{189000}-\frac{5939 u^{8}}{15240960000}
\end{aligned}
$$




$$
\begin{aligned}
& \gamma_{n+2}=-\frac{(u \cos (u / 2)-2 \sin (u / 2)) \sin (u / 2)}{u(u \cos u-\sin u)} \\
& =-\frac{1}{8}-\frac{u^{2}}{240}-\frac{13 u^{4}}{67200}-\frac{19 u^{6}}{2016000}-\frac{12979 u^{8}}{27941760000} \\
& \quad-\frac{83437 u^{10}}{3632428800000}+O\left(h^{11}\right), \\
& \widehat{\beta}_{0,0} \\
& =-\frac{\csc (u / 2)^{2}\left(-2-u^{2}+\left(4+3 u^{2}\right) \cos u-2 \cos 2 u-2 u \sin 2 u\right)}{8 u(u \cos u-\sin u)} \\
& =-\frac{17}{48}-\frac{u^{2}}{72}-\frac{251 u^{4}}{403200}-\frac{169 u^{6}}{6048000}-\frac{213203 u^{8}}{167650560000} \\
& \quad-\frac{161023 u^{10}}{2724321600000}+O\left(h^{11}\right), \\
& \widehat{\beta}_{1,0} \\
& =\frac{\csc (u / 2)^{2}\left(2+u^{2}+\left(-2+3 u^{2}\right) \cos 2 u+4 u \sin u-6 u \sin 2 u\right)}{(8 u(u \cos u-\sin u))}
\end{aligned}
$$

$$
\begin{aligned}
= & -\frac{11}{12}+\frac{17 u^{2}}{720}+\frac{53 u^{4}}{50400}+\frac{281 u^{6}}{6048000}+\frac{87133 u^{8}}{41912640000} \\
& +\frac{1037873 u^{10}}{10897286400000}+O\left(h^{11}\right), \\
\widehat{\beta}_{2,0} & =-\frac{\csc (u / 2)^{2}\left(4+\left(-4+u^{2}\right) \cos u+u^{2} \cos 2 u-2 u \sin 2 u\right)}{8 u(u \cos u-\sin u)} \\
= & \frac{13}{48}-\frac{7 u^{2}}{720}-\frac{173 u^{4}}{403200}-\frac{u^{6}}{54000}-\frac{135329 u^{8}}{167650560000} \\
& -\frac{393781 u^{10}}{10897286400000}+O\left(h^{11}\right), \\
\widehat{\gamma}_{n+2,0} & =-\frac{(u \cos (u / 2)-2 \sin (u / 2)) \sin (u / 2)}{u(u \cos u-\sin u)} \\
= & -\frac{1}{8}-\frac{u^{2}}{240}-\frac{13 u^{4}}{67200}-\frac{19 u^{6}}{2016000}-\frac{12979 u^{8}}{27941760000} \\
& -\frac{83437 u^{10}}{3632428800000}+O\left(h^{11}\right) .
\end{aligned}
$$

Case $k=3$. The coefficients of the main method (10) and the complimentary methods (11) are, respectively, defined by

$$
\begin{aligned}
& \beta_{0}=\frac{\left(\csc (u / 4)^{4}\left(-42-5 u^{2}-8\left(-6+u^{2}\right) \cos u+\left(-6+u^{2}\right) \cos 2 u+32 u \sin u-4 u \sin 2 u\right)\right)}{(96 u(u+2 u \cos u-3 \sin u))} \\
& =\frac{7}{1080}+\frac{163 u^{2}}{151200}+\frac{1529 u^{4}}{13608000}+\frac{1203457 u^{6}}{125737920000}+\frac{5143273 u^{8}}{7005398400000}+\frac{364730953 u^{10}}{6865290432000000}+O\left(h^{11}\right) \text {, } \\
& \beta_{1}=-\frac{\left(\csc (u / 4)^{4}\left(-102-16 u^{2}-3\left(-34+7 u^{2}\right) \cos u+6 \cos 2 u-6 \cos 3 u+u^{2} \cos 3 u+88 u \sin u-2 u \sin 2 u-4 u \sin 3 u\right)\right)}{(96 u(u+2 u \cos u-3 \sin u))} \\
& =-\frac{1}{20}-\frac{11 u^{2}}{8400}-\frac{173 u^{4}}{756000}-\frac{153049 u^{6}}{6985440000}-\frac{4786027 u^{8}}{2724321600000}-\frac{422813 u^{10}}{3259872000000}+O\left(h^{11}\right), \\
& \beta_{2}=\frac{\left(\csc (u / 4)^{4}\left(-30-23 u^{2}-24 \cos u+\left(78-21 u^{2}\right) \cos 2 u-24 \cos 3 u+8 u^{2} \cos 3 u+34 u \sin u+52 u \sin 2 u-22 u \sin 3 u\right)\right)}{(96 u(u+2 u \cos u-3 \sin u))} \\
& =\frac{19}{40}-\frac{u^{2}}{224}-\frac{29 u^{4}}{302400}+\frac{4187 u^{6}}{2794176000}+\frac{41551 u^{8}}{99066240000}+\frac{6204403 u^{10}}{152562009600000}+O\left(h^{11}\right), \\
& \beta_{3}=-\frac{\left(\csc (u / 4)^{4}\left(30-\left(78+23 u^{2}\right) \cos u+2\left(33+8 u^{2}\right) \cos 2 u-18 \cos 3 u-5 u^{2} \cos 3 u+68 u \sin u-22 u \sin 2 u\right)\right)}{(96 u(u+2 u \cos u-3 \sin u))} \\
& =\frac{307}{540}+\frac{71 u^{2}}{15120}+\frac{289 u^{4}}{1360800}+\frac{12391 u^{6}}{1143072000}+\frac{2957783 u^{8}}{4903778880000}+\frac{24651509 u^{10}}{686529043200000}+O\left(h^{11}\right), \\
& \gamma_{n+3}=\frac{\csc (u / 2)(7 u \cos (u / 2)+5 u \cos (3 u / 2)+12(\sin (u / 2)-\sin (3 u / 2)))}{12 u(u+2 u \cos u-3 \sin u)} \\
& =-\frac{19}{180}-\frac{97 u^{2}}{25200}-\frac{491 u^{4}}{2268000}-\frac{285163 u^{6}}{20956320000}-\frac{7286749 u^{8}}{8172964800000}-\frac{67916227 u^{10}}{1144215072000000}+O\left(h^{11}\right), \\
& \widehat{\beta}_{0,0}=\frac{\csc (u / 2)^{4}\left(-12+2 u^{2}+\left(9-4 u^{2}\right) \cos u+2\left(6+7 u^{2}\right) \cos (2 u)-9 \cos (3 u)+34 u \sin u-20 u \sin (2 u)-6 u \sin (3 u)\right)}{(48 u(u+2 u \cos u-3 \sin u))} \\
& =-\frac{43}{135}-\frac{43 u^{2}}{4725}-\frac{193 u^{4}}{850500}-\frac{2561 u^{6}}{1964655000}+\frac{1107763 u^{8}}{3064861800000}+\frac{8005337 u^{10}}{214540326000000}+O\left(h^{11}\right), \\
& \widehat{\beta}_{1,0} \\
& =\frac{-\left(\csc (u / 2)^{4}\left(-15-4 u^{2}+3\left(4+5 u^{2}\right) \cos u+15 \cos (2 u)-12 \cos (3 u)+7 u^{2} \cos (3 u)-5 u \sin u+13 u \sin (2 u)-19 u \sin (3 u)\right)\right)}{(24 u(u+2 u \cos u-3 \sin u))}
\end{aligned}
$$




$$
\begin{aligned}
& =-\frac{7}{5}+\frac{29 u^{2}}{1050}+\frac{139 u^{4}}{189000}+\frac{3683 u^{6}}{436590000}-\frac{516289 u^{8}}{681080400000}-\frac{473779 u^{10}}{5297292000000}+O\left(h^{11}\right), \\
& \widehat{\beta}_{2,0} \\
& =\frac{\csc (u / 2)^{4}\left(-12+2 u^{2}+15 \cos u+6\left(2+5 u^{2}\right) \cos (2 u)-15 \cos (3 u)+4 u^{2} \cos (3 u)+44 u \sin u-28 u \sin (2 u)-20 u \sin (3 u)\right)}{(48 u(u+2 u \cos u-3 \sin u))} \\
& =-\frac{1}{5}-\frac{u^{2}}{35}-\frac{17 u^{4}}{18900}-\frac{961 u^{6}}{43659000}-\frac{1367 u^{8}}{6191640000}+\frac{104537 u^{10}}{4767562800000}+O\left(h^{11}\right) \\
& \widehat{\beta}_{3,0}=\frac{-\left(\csc (u / 2)^{4}\left(3+u^{2} \cos u+\left(-3+4 u^{2}\right) \cos (2 u)+u^{2} \cos (3 u)-u \sin u-u \sin (2 u)-3 u \sin (3 u)\right)\right)}{(24 u(u+2 u \cos u-3 \sin u))} \\
& =-\frac{11}{135}+\frac{19 u^{2}}{1890}+\frac{19 u^{4}}{48600}+\frac{1063 u^{6}}{71442000}+\frac{756881 u^{8}}{1225944720000}+\frac{2591419 u^{10}}{85816130400000}+O\left(h^{11}\right), \\
& \widehat{\gamma}_{n+3,0}=-\frac{2 \cot (u / 2)(2 u+u \cos u-3 \sin u)}{3 u(u+2 u \cos u-3 \sin u)} \\
& =\frac{2}{45}-\frac{u^{2}}{1575}-\frac{31 u^{4}}{283500}-\frac{5927 u^{6}}{654885000}-\frac{95237 u^{8}}{145945800000}-\frac{3218641 u^{10}}{71513442000000}+O\left(h^{11}\right), \\
& \widehat{\beta}_{0,1}=\frac{\csc (u / 2)^{4}\left(-30-u^{2}+8\left(6+u^{2}\right) \cos u+\left(-18+5 u^{2}\right) \cos (2 u)+16 u \sin u-20 u \sin (2 u)\right)}{(96 u(u+2 u \cos u-3 \sin u))} \\
& =\frac{23}{1080}+\frac{467 u^{2}}{151200}+\frac{3841 u^{4}}{13608000}+\frac{2702753 u^{6}}{125737920000}+\frac{74202119 u^{8}}{49037788800000}+\frac{707967737 u^{10}}{6865290432000000}+O\left(h^{11}\right) \text {, } \\
& \widehat{\beta}_{1,1}=\frac{-\left(\csc (u / 2)^{4}\left(-54-8 u^{2}+\left(96+39 u^{2}\right) \cos u-42 \cos (2 u)+5 u^{2} \cos (3 u)-4 u \sin u-22 u \sin (2 u)-8 u \sin (3 u)\right)\right)}{(96 u(u+2 u \cos u-3 \sin u))} \\
& =-\frac{9}{20}-\frac{59 u^{2}}{8400}-\frac{517 u^{4}}{756000}-\frac{371321 u^{6}}{6985440000}-\frac{10263683 u^{8}}{2724321600000}-\frac{32697403 u^{10}}{127135008000000}+O\left(h^{11}\right), \\
& \widehat{\beta}_{2,1}=\frac{\csc (u / 2)^{4}\left(54+5 u^{2}-48 \cos u+\left(-6+39 u^{2}\right) \cos (2 u)-8 u^{2} \cos (3 u)+50 u \sin u-76 u \sin (2 u)+10 u \sin (3 u)\right)}{(96 u(u+2 u \cos u-3 \sin u))} \\
& =-\frac{29}{40}+\frac{u^{2}}{3360}+\frac{59 u^{4}}{302400}+\frac{53563 u^{6}}{2794176000}+\frac{1581469 u^{8}}{1089728640000}+\frac{1395817 u^{10}}{13869273600000}+O\left(h^{11}\right) \\
& \widehat{\beta}_{3,1}=\frac{-\left(\csc (u / 2)^{4}\left(78+\left(-96+5 u^{2}\right) \cos u+2\left(9+4 u^{2}\right) \cos (2 u)-u^{2} \cos (3 u)-20 u \sin u-2 u \sin (2 u)\right)\right)}{(96 u(u+2 u \cos u-3 \sin u))} \\
& =\frac{83}{540}+\frac{11 u^{2}}{3024}+\frac{281 u^{4}}{1360800}+\frac{14279 u^{6}}{1143072000}+\frac{3937807 u^{8}}{4903778880000}+\frac{36676261 u^{10}}{686529043200000}+O\left(h^{11}\right), \\
& \widehat{\gamma}_{n+3,1}=\frac{\csc (u / 2)(-13 u \cos (u / 2)+u \cos ((3 u) / 2)+24 \sin (u / 2))}{12 u(u+2 u \cos u-3 \sin u)} \\
& =-\frac{11}{180}-\frac{113 u^{2}}{25200}-\frac{739 u^{4}}{2268000}-\frac{474827 u^{6}}{20956320000}-\frac{12620021 u^{8}}{8172964800000}-\frac{119414483 u^{10}}{1144215072000000}+O\left(h^{11}\right) .
\end{aligned}
$$

Case $k=4$. The coefficients of the main method (10) and the complimentary methods (11) are, respectively, defined by

$$
\begin{aligned}
\beta_{0} & =\frac{\left(N_{\beta_{0}}\right)}{(384 u(6 u \cos (2 u)+16 \sin u-11 \sin (2 u)))} \\
& =-\frac{17}{5760}-\frac{251 u^{2}}{483840}-\frac{2003 u^{4}}{33868800}
\end{aligned}
$$

$$
\begin{aligned}
& -\frac{354659 u^{6}}{62589542400}-\frac{51104113 u^{8}}{102521670451200} \\
& -\frac{600902219 u^{10}}{14353033863168000}+O\left(h^{11}\right)
\end{aligned}
$$$$
\beta_{1}=\frac{\left(N_{\beta_{1}}\right)}{(384 u(6 u \cos (2 u)+16 \sin u-11 \sin (2 u)))}
$$ 


$$
\begin{aligned}
& =\frac{1}{45}+\frac{29 u^{2}}{30240}+\frac{181 u^{4}}{1058400}+\frac{70811 u^{6}}{3911846400} \\
& +\frac{2645263 u^{8}}{1601901100800}+\frac{9724577 u^{10}}{69004970496000} \\
& +O\left(h^{11}\right) \text {, } \\
& \beta_{2}=\frac{\left(N_{\beta_{2}}\right)}{(192 u(6 u \cos (2 u)+16 \sin u-11 \sin (2 u)))} \\
& =-\frac{41}{480}+\frac{11 u^{2}}{5760}-\frac{169 u^{4}}{2822400}-\frac{70207 u^{6}}{5215795200} \\
& -\frac{12382199 u^{8}}{8543472537600}-\frac{14328617 u^{10}}{108735105024000} \\
& +O\left(h^{11}\right) \text {, } \\
& \beta_{3}=\frac{\left(N_{\beta_{3}}\right)}{(384 u(6 u \cos (2 u)+16 \sin u-11 \sin (2 u)))} \\
& =\frac{47}{90}-\frac{241 u^{2}}{30240}-\frac{187 u^{4}}{529200}-\frac{72019 u^{6}}{3911846400} \\
& -\frac{3489379 u^{8}}{3203802201600}-\frac{64028929 u^{10}}{897064616448000} \\
& +O\left(h^{11}\right) \text {, } \\
& \beta_{4}=\frac{\left(N_{\beta_{4}}\right)}{(384 u(6 u \cos (2 u)+16 \sin u-11 \sin (2 u)))} \\
& =\frac{3133}{5760}+\frac{2719 u^{2}}{483840}+\frac{10207 u^{4}}{33868800}+\frac{1216471 u^{6}}{62589542400} \\
& +\frac{142053797 u^{8}}{102521670451200}+\frac{1494030511 u^{10}}{14353033863168000} \\
& +O\left(h^{11}\right) \text {, } \\
& \gamma_{n+4}=\frac{\left(N_{\gamma_{n+4}}\right)}{2 u(6 u \cos (2 u)+16 \sin u-11 \sin (2 u))} \\
& =-\frac{3}{32}-\frac{3 u^{2}}{896}-\frac{37 u^{4}}{188160}-\frac{529 u^{6}}{38635520} \\
& -\frac{195109 u^{8}}{189854945280}-\frac{6348281 u^{10}}{79739077017600} \\
& +O\left(h^{11}\right)
\end{aligned}
$$

with $N_{\beta_{i}}, i=0,1, \ldots, 4$, and $N_{\gamma_{n+4}}$ defined in part A of Appendix 1 of the supplementary material (see Supplementary Material available online at http://dx.doi.org/10.1155/2015/343295). Consider

$$
\begin{aligned}
\widehat{\beta}_{0,0} & =\frac{N_{\widehat{\beta}_{0,0}}}{(128 u(6 u \cos (2 u)+16 \sin u-11 \sin (2 u)))} \\
& =-\frac{201}{640}-\frac{23 u^{2}}{2560}-\frac{1019 u^{4}}{3763200}-\frac{20569 u^{6}}{2318131200}
\end{aligned}
$$

$$
\begin{aligned}
& -\frac{1712483 u^{8}}{3797098905600}-\frac{6213083 u^{10}}{177197948928000} \\
& +O\left(h^{11}\right)
\end{aligned}
$$$$
\widehat{\beta}_{1,0}=\frac{N_{\widehat{\beta}_{1,0}}}{(128 u(6 u \cos (2 u)+16 \sin u-11 \sin (2 u)))}
$$$$
=-\frac{7}{5}+\frac{39 u^{2}}{1120}+\frac{359 u^{4}}{352800}+\frac{4481 u^{6}}{144883200}
$$$$
+\frac{260119 u^{8}}{177989011200}+\frac{34002959 u^{10}}{299021538816000}
$$$$
+O\left(h^{11}\right) \text {, }
$$$$
\widehat{\beta}_{2,0}=\frac{N_{\widehat{\beta}_{2,0}}}{(64 u(6 u \cos (2 u)+16 \sin u-11 \sin (2 u)))}
$$$$
=-\frac{99}{160}-\frac{219 u^{2}}{4480}-\frac{417 u^{4}}{313600}-\frac{6317 u^{6}}{193177600}
$$$$
-\frac{40941 u^{8}}{35158323200}-\frac{12088171 u^{10}}{132898461696000}
$$$$
+O\left(h^{11}\right) \text {, }
$$$$
\widehat{\beta}_{3,0}=\frac{N_{\widehat{\beta}_{3,0}}}{(128 u(6 u \cos (2 u)+16 \sin u-11 \sin (2 u)))}
$$$$
=-\frac{9}{10}+\frac{29 u^{2}}{1120}+\frac{29 u^{4}}{58800}-\frac{809 u^{6}}{144883200}
$$$$
-\frac{151769 u^{8}}{118659340800}-\frac{3275539 u^{10}}{33224615424000}
$$$$
+O\left(h^{11}\right) \text {, }
$$$$
\widehat{\beta}_{4,0}=\frac{N_{\widehat{\beta}_{4,0}}}{(128 u(6 u \cos (2 u)+16 \sin u-11 \sin (2 u)))}
$$$$
=\frac{149}{640}-\frac{51 u^{2}}{17920}+\frac{1013 u^{4}}{11289600}+\frac{37621 u^{6}}{2318131200}
$$$$
+\frac{16324541 u^{8}}{11391296716800}+\frac{530557669 u^{10}}{4784344621056000}
$$$$
+O\left(h^{11}\right) \text {, }
$$

$$
\begin{aligned}
\widehat{\gamma}_{n+4,0}= & \frac{N_{\widehat{\gamma}_{n+4,0}}}{2 u(6 u \cos (2 u)+16 \sin u-11 \sin (2 u))} \\
= & -\frac{3}{32}-\frac{3 u^{2}}{896}-\frac{37 u^{4}}{188160}-\frac{529 u^{6}}{38635520} \\
& -\frac{195109 u^{8}}{189854945280}-\frac{6348281 u^{10}}{79739077017600} \\
& +O\left(h^{11}\right)
\end{aligned}
$$


with $N_{\widehat{\beta}_{i, 0}}, i=0,1, \ldots, 4$, and $N_{\widehat{\gamma}_{n+4,0}}$ defined in part B of Appendix 1 of the supplementary material. Consider

$$
\begin{aligned}
\widehat{\beta}_{0,1}= & \frac{N_{\widehat{\beta}_{0,1}}}{(48 u(6 u \cos (2 u)+16 \sin u-11 \sin (2 u)))} \\
= & \frac{1}{90}+\frac{u^{2}}{756}+\frac{u^{4}}{10800}+\frac{u^{6}}{199584} \\
& +\frac{691 u^{8}}{2971987200}+\frac{u^{10}}{102643200}+O\left(h^{11}\right), \\
\widehat{\beta}_{1,1}= & \frac{N_{\hat{\beta}_{1,1}}}{(48 u(6 u \cos (2 u)+16 \sin u-11 \sin (2 u)))} \\
= & -\frac{17}{45}-\frac{u^{2}}{189}-\frac{u^{4}}{2700}-\frac{u^{6}}{49896}-\frac{691 u^{8}}{742996800} \\
& -\frac{u^{10}}{25660800}+O\left(h^{11}\right), \\
\widehat{\beta}_{2,1}= & \frac{N_{\hat{\beta}_{2,1}}}{(24 u(6 u \cos (2 u)+16 \sin u-11 \sin (2 u)))} \\
= & -\frac{19}{15}+\frac{u^{2}}{126}+\frac{u^{4}}{1800}+\frac{u^{6}}{33264}+\frac{691 u^{8}}{495331200} \\
& +\frac{u^{10}}{17107200}+O\left(h^{11}\right), \\
\widehat{\gamma}_{n+4,1}= & 0 \frac{691 u^{8}}{2971987200}+\frac{u^{10}}{102643200}+O\left(h^{11}\right), \\
\widehat{\beta}_{3,1}= & \frac{N_{\widehat{\beta}_{3,1}}}{(48 u(6 u \cos (2 u)+16 \sin u-11 \sin (2 u)))} \\
= & -\frac{17}{45}-\frac{u^{2}}{189}-\frac{u^{4}}{25660000}-\frac{u^{6}}{49896}-\frac{691 u^{8}}{742996800} \\
N_{\hat{\beta}_{4,1}} & +\frac{u^{2}}{756}+\frac{u^{4}}{10800}+\frac{u^{6}}{199584} \\
& \\
& \\
&
\end{aligned}
$$

with $N_{\widehat{\beta}_{i, 1}}, i=0,1, \ldots, 4$, defined in part C of Appendix 1 of the supplementary material. Consider

$$
\begin{aligned}
\widehat{\beta}_{0,2} & =\frac{N_{\widehat{\beta}_{0,2}}}{(384 u(6 u \cos (2 u)+16 \sin u-11 \sin (2 u)))} \\
& =-\frac{11}{1920}-\frac{169 u^{2}}{161280}-\frac{12281 u^{4}}{101606400}
\end{aligned}
$$

$$
\begin{aligned}
& -\frac{2158889 u^{6}}{187768627200}-\frac{7853429 u^{8}}{7886282342400} \\
& -\frac{272561573 u^{10}}{3312238583808000}+O\left(h^{11}\right) \text {, } \\
& \widehat{\beta}_{1,2}=\frac{N_{\widehat{\beta}_{1,2}}}{(384 u(6 u \cos (2 u)+16 \sin u-11 \sin (2 u)))} \\
& =\frac{7}{135}+\frac{31 u^{2}}{10080}+\frac{3701 u^{4}}{9525600}+\frac{1346483 u^{6}}{35206617600} \\
& +\frac{16159921 u^{8}}{4805703302400}+\frac{752918951 u^{10}}{2691193849344000} \\
& +O\left(h^{11}\right) \text {, } \\
& \widehat{\beta}_{2,2}=\frac{N_{\widehat{\beta}_{2,2}}}{(192 u(6 u \cos (2 u)+16 \sin u-11 \sin (2 u)))} \\
& =-\frac{83}{160}-\frac{17 u^{2}}{13440}-\frac{841 u^{4}}{2822400}-\frac{534077 u^{6}}{15647385600} \\
& -\frac{9061597 u^{8}}{2847824179200}-\frac{974213257 u^{10}}{3588258465792000} \\
& +O\left(h^{11}\right) \text {, } \\
& \widehat{\beta}_{3,2}=\frac{N_{\widehat{\beta}_{3,2}}}{(384 u(6 u \cos (2 u)+16 \sin u-11 \sin (2 u)))} \\
& =-\frac{19}{30}-\frac{59 u^{2}}{10080}-\frac{589 u^{4}}{1587600}-\frac{278329 u^{6}}{11735539200} \\
& -\frac{5135051 u^{8}}{3203802201600}-\frac{28211849 u^{10}}{244653986304000} \\
& +O\left(h^{11}\right) \text {, } \\
& \widehat{\beta}_{4,2}=\frac{N_{\widehat{\beta}_{4,2}}}{(384 u(6 u \cos (2 u)+16 \sin u-11 \sin (2 u)))} \\
& =\frac{1831}{17280}+\frac{821 u^{2}}{161280}+\frac{122327 u^{4}}{304819200} \\
& +\frac{17519503 u^{6}}{563305881600}+\frac{743666159 u^{8}}{307565011353600} \\
& +\frac{8152441741 u^{10}}{43059101589504000}+O\left(h^{11}\right) \text {, } \\
& \widehat{\gamma}_{n+4,2}=\frac{N_{\widehat{\gamma}_{n+4,2}}}{2 u(6 u \cos (2 u)+16 \sin u-11 \sin (2 u))} \\
& =-\frac{11}{288}-\frac{3 u^{2}}{896}-\frac{1447 u^{4}}{5080320}-\frac{218147 u^{6}}{9388431360} \\
& -\frac{9544999 u^{8}}{5126083522560}-\frac{11813881 u^{10}}{79739077017600} \\
& +O\left(h^{11}\right)
\end{aligned}
$$


with $N_{\widehat{\beta}_{i, 2}}, i=0,1, \ldots, 4$, and $N_{\widehat{\gamma}_{n+4,2}}$ defined in part $\mathrm{D}$ of Appendix 1 of the supplementary material.

2.3. Block Specification and Implementation of the Methods. We consider a general procedure for the block implementation of the methods in matrix form (see Fatunla [26]). First we define the following vectors:

$$
\begin{aligned}
Y_{\mu+1} & =\left[y_{n+1}, y_{n+2}, y_{n+3}, \ldots, y_{n+k}\right]^{T}, \\
Y_{\mu} & =\left[y_{n-k+1}, y_{n-k+2}, y_{n-k+3}, \ldots, y_{n}\right]^{T}, \\
F_{\mu+1} & =\left[f_{n+1}, f_{n+2}, f_{n+3}, \ldots, f_{n+k}\right]^{T}, \\
F_{\mu} & =\left[f_{n-k+1}, f_{n-k+2}, f_{n-k+3}, \ldots, f_{n}\right]^{T}, \\
G_{\mu+1} & =\left[g_{n+1}, g_{n+2}, g_{n+3}, \ldots, g_{n+k}\right]^{T},
\end{aligned}
$$

where $y_{n+j}=y\left(x_{n}+j h\right), f_{n+j}=f\left(x_{n}+j h, y\left(x_{n}+j h\right)\right)$, and $g_{n+j}=\left.(d f(x, y(x)) / d x)\right|_{y_{n+j}} ^{x_{n+j}}$. The integration on the entire block will be compactly written as

$$
\begin{array}{r}
A_{1} Y_{\mu+1}=A_{0} Y_{\mu}+h B_{0} F_{\mu}+h B_{1} F_{\mu+1}+h^{2} C_{1} G_{\mu+1}, \\
\mu=0,1, \ldots,
\end{array}
$$

which forms a nonlinear equation because of the implicit nature, and hence we employ the Newton iteration for the evaluation of the approximate solutions. We use Newton's approach for the implementation of implicit schemes to get the following solution of the block:

$$
\begin{aligned}
& Y_{\mu+1}^{(i+1)}=Y_{\mu+1}^{(i)}-\left(A_{1}-h B_{1} \frac{\partial F_{\mu+1}}{\partial Y}-h^{2} C_{1} \frac{\partial G_{\mu+1}}{\partial Y}\right)^{-1} \\
& \cdot\left(A_{1} Y_{\mu+1}\right. \\
& \left.-A_{0} Y_{\mu}-h B_{0} F_{\mu}-h B_{1} F_{\mu+1}-h^{2} C_{1} G_{\mu+1}\right) .
\end{aligned}
$$

The $k \times k$ matrices $A_{0}, A_{1}, B_{0}, B_{1}$, and $C_{1}$ are defined as follows.

Case $k=2$. Consider

$$
\begin{aligned}
A_{0} & =\left(\begin{array}{cc}
0 & -1 \\
0 & 0
\end{array}\right), \\
A_{1} & =\left(\begin{array}{cc}
-1 & 0 \\
-1 & 1
\end{array}\right), \\
B_{0} & =\left(\begin{array}{ll}
0 & \widehat{\beta}_{0,0} \\
0 & \beta_{0}
\end{array}\right), \\
B_{1} & =\left(\begin{array}{cc}
\widehat{\beta}_{1,0} & \widehat{\beta}_{2,0} \\
\beta_{1} & \beta_{2}
\end{array}\right), \\
C_{1} & =\left(\begin{array}{ll}
0 & \widehat{\gamma}_{n+2,0} \\
0 & \gamma_{n+2}
\end{array}\right)
\end{aligned}
$$

with $\beta_{i}, \widehat{\beta}_{i, j}, \gamma_{n+k}, \widehat{\gamma}_{n+k, j}, j=0, i=1,2$, defined in methods (13) and (14).

Case $k=3$. Consider

$$
\begin{aligned}
& A_{0}=\left(\begin{array}{ccc}
0 & 0 & 0 \\
0 & 0 & -1 \\
0 & 0 & 0
\end{array}\right), \\
& A_{1}=\left(\begin{array}{ccc}
1 & -1 & 0 \\
0 & -1 & 0 \\
0 & -1 & 1
\end{array}\right) \text {, } \\
& B_{0}=\left(\begin{array}{ccc}
0 & 0 & \widehat{\beta}_{0,1} \\
0 & 0 & \widehat{\beta}_{0,0} \\
0 & 0 & \beta_{0}
\end{array}\right) \text {, } \\
& B_{1}=\left(\begin{array}{ccc}
\hat{\beta}_{1,1} & \widehat{\beta}_{2,1} & \widehat{\beta}_{3,1} \\
\widehat{\beta}_{1,0} & \widehat{\beta}_{2,0} & \widehat{\beta}_{3,0} \\
\beta_{1} & \beta_{2} & \beta_{3}
\end{array}\right) \text {, } \\
& C_{1}=\left(\begin{array}{ccc}
0 & 0 & \widehat{\gamma}_{n+3,1} \\
0 & 0 & \widehat{\gamma}_{n+3,0} \\
0 & 0 & \gamma_{n+3}
\end{array}\right),
\end{aligned}
$$

with $\beta_{i}, \widehat{\beta}_{i, j}, \gamma_{n+k}, \widehat{\gamma}_{n+k, j}, j=0,1, i=1,2,3$, defined in methods (15), (16), and (17).

Case $k=4$. Consider

$$
\begin{aligned}
A_{0} & =\left(\begin{array}{llll}
0 & 0 & 0 & 0 \\
0 & 0 & 0 & 0 \\
0 & 0 & 0 & -1 \\
0 & 0 & 0 & 0
\end{array}\right), \\
A_{1} & =\left(\begin{array}{cccc}
1 & 0 & -1 & 0 \\
0 & 1 & -1 & 0 \\
0 & 0 & -1 & 0 \\
0 & 0 & -1 & 1
\end{array}\right), \\
B_{0} & =\left(\begin{array}{llll}
0 & 0 & 0 & \hat{\beta}_{0,1} \\
0 & 0 & 0 & \hat{\beta}_{0,2} \\
0 & 0 & 0 & \widehat{\beta}_{0,0} \\
0 & 0 & 0 & \beta_{0}
\end{array}\right),
\end{aligned}
$$




$$
\begin{aligned}
B_{1} & =\left(\begin{array}{llll}
\widehat{\beta}_{1,1} & \widehat{\beta}_{2,1} & \widehat{\beta}_{3,1} & \widehat{\beta}_{4,1} \\
\widehat{\beta}_{1,2} & \widehat{\beta}_{2,2} & \widehat{\beta}_{3,2} & \widehat{\beta}_{4,2} \\
\widehat{\beta}_{1,0} & \widehat{\beta}_{2,0} & \widehat{\beta}_{3,0} & \widehat{\beta}_{4,0} \\
\beta_{1} & \beta_{2} & \beta_{3} & \beta_{4}
\end{array}\right), \\
C_{1} & =\left(\begin{array}{llll}
0 & 0 & 0 & \widehat{\gamma}_{n+4,1} \\
0 & 0 & 0 & \widehat{\gamma}_{n+4,2} \\
0 & 0 & 0 & \widehat{\gamma}_{n+4,0} \\
0 & 0 & 0 & \gamma_{n+4}
\end{array}\right)
\end{aligned}
$$

with $\beta_{i}, \widehat{\beta}_{i, j}, \gamma_{n+k}, \widehat{\gamma}_{n+k, j}, i=1,2, \ldots, k$ and $j=0,1,2, \ldots, k-2$, defined in methods (18) through (21).

\section{Error Analysis and Stability}

3.1. Local Truncation Error (LTE). Suppose that method (10) is associated with a linear difference operator:

$$
\begin{gathered}
L\left[y\left(x_{n}: h\right)\right]=[y(x+k h)-y(x+(k-1) h) \\
\left.-h \sum_{j=0}^{k} \beta_{j} y^{\prime}(x+j h)-h^{2} \gamma_{n+k} y^{\prime \prime}(x+k h)\right],
\end{gathered}
$$

where $y(x)$ is an arbitrary smooth function. Then $L\left[y\left(x_{n} ; h\right)\right]$ is called the local truncation error at $x_{n+k}$ if $y$ represents a solution of the IVP (2). By a Taylor series expansion of $y(x+$ $j h), y^{\prime}(x+j h)$, and $y^{\prime \prime}(x+j h), j=0,1,2, \ldots, k$, we have

$$
\begin{aligned}
L\left[y\left(x_{n} ; h\right)\right]= & C_{0} y(x)+C_{1} h y^{\prime}(x)+C_{2} \frac{h^{2}}{2 !} y^{\prime \prime}(x) \\
& +\cdots+C_{q} \frac{h^{q}}{q !} y^{(q)}(x)+\cdots,
\end{aligned}
$$

where $C_{0}=\sum_{j=0}^{k} \alpha_{j}, C_{1}=1-\sum_{j=0}^{k} \beta_{j}$, and $C_{2}=(1 / 2 !)(-(k-$ $\left.1)^{2}+k^{2}\right)-\sum_{j=0}^{k} j \beta_{j}-\gamma_{n+k}, \ldots, C_{q}=(1 / q !)\left(-(k-1)^{2}+k^{q}\right)-$ $(1 /(q-1) !) \sum_{j=0}^{k} j^{q-1} \beta_{j}-(1 /(q-2) !) k^{q-2} \gamma_{n+k}$.

Method (10) is said to be of order $p$ if $C_{0}=C_{1}=\cdots=$ $C_{p}=0, C_{p+1} \neq 0$ (see [3]).

Theorem 3. The k-step method (10) ESDM has a local truncation error (LTE) of

$$
C_{k+3} h^{k+3}\left(w^{2} y^{(k+1)}\left(x_{n}\right)+y^{(k+3)}\left(x_{n}\right)\right)+O\left(h^{k+4}\right) .
$$

Proof. We consider a Taylor series expansion of $y_{n+j}, y(x+$ $j h), y_{n+j}^{\prime}, y^{\prime}(x+j h), y_{n+j}^{\prime \prime}, y^{\prime \prime}(x+j h)$ and assume that $y\left(x_{n+j}\right)=y_{n+j}, y^{\prime}\left(x_{n+j}\right)=f_{n+j}, y^{\prime \prime}\left(x_{n+k}\right)=g_{n+k}$. Then by
TABLE 1: The local truncation error for various cases.

\begin{tabular}{lccc}
\hline Case $(k)$ & Method & Order $(p)$ & Error constant $\left(C_{p+1}\right)$ \\
\hline 1 & $(12)$ & 3 & $1 / 7$ \\
\hline 2 & $(13)$ & 4 & $7 / 1440$ \\
& $(14)$ & 4 & $23 / 1440$ \\
\hline \multirow{3}{*}{3} & $(15)$ & 5 & $17 / 7200$ \\
& $(16)$ & 5 & $-2 / 225$ \\
& $(17)$ & 5 & $11 / 2400$ \\
\hline \multirow{3}{*}{4} & $(18)$ & 6 & $41 / 30240$ \\
& $(19)$ & 6 & $11 / 1120$ \\
& $(20)$ & 6 & $-1 / 756$ \\
& $(21)$ & 6 & $19 / 10080$ \\
\hline
\end{tabular}

substituting these into method (10) and simplifying we get that

$$
\begin{aligned}
\mathrm{LTE}= & y\left(x_{n+k}\right)-y_{n+k} \\
= & C_{k+3} h^{k+3}\left(w^{2} y^{(k+1)}\left(x_{n}\right)+y^{(k+3)}\left(x_{n}\right)\right) \\
& +O\left(h^{k+4}\right),
\end{aligned}
$$

where the values of $C_{k+3}$ are given in Table 1 .

Define the local truncation error of (23) as follows:

$$
\begin{aligned}
L & {[Z(x) ; h] } \\
& =\mathbf{Z}_{\mu+1}-\left[A \mathbf{Z}_{\mu}+h B \overline{\mathbf{F}}_{\mu}+h D \overline{\mathbf{F}}_{\mu+1}+h^{2} C \overline{\mathbf{G}}_{\mu+1}\right],
\end{aligned}
$$

where

$$
\begin{aligned}
& \mathbf{Z}_{\mu+1}=\left[y\left(x_{n+1}\right), y\left(x_{n+2}\right), y\left(x_{n+3}\right), \ldots, y\left(x_{n+k}\right)\right]^{T}, \\
& \mathbf{Z}_{\mu}=\left[y\left(x_{n-k+1}\right), y\left(x_{n-k+2}\right), y\left(x_{n-k+3}\right), \ldots, y\left(x_{n}\right)\right]^{T}, \\
& \overline{\mathbf{F}}_{\mu+1}=\left[f\left(x_{n+1}\right), f\left(x_{n+2}\right), f\left(x_{n+3}\right), \ldots, f\left(x_{n+k}\right)\right]^{T}, \\
& \overline{\mathbf{F}}_{\mu}=\left[f\left(x_{n-k+1}\right), f\left(x_{n-k+2}\right), f\left(x_{n-k+3}\right), \ldots,\right. \\
& \left.\quad f\left(x_{n}\right)\right]^{T}, \\
& \overline{\mathbf{G}}_{\mu+1}=\left[g\left(x_{n+1}\right), g\left(x_{n+2}\right), g\left(x_{n+3}\right), \ldots, g\left(x_{n+k}\right)\right]^{T}, \\
& L[Z(x) ; h]=\left(L_{1}[Z(x) ; h], L_{2}[Z(x) ; h],\right. \\
& \left.\quad L_{3}[Z(x) ; h], \ldots, L_{k}[Z(x) ; h]\right)^{T}
\end{aligned}
$$

a linear difference operator. Assuming that $Z(x)$ is sufficiently differentiable, we can expand the terms in (23) as a Taylor series about $x$ to obtain the expression for the local truncation error

$$
\begin{aligned}
L[Z(x) ; h]= & C_{0} Z(x)+C_{1} h Z^{\prime}(x)+\cdots \\
& +C_{q} h^{q} Z^{q}(x)+\cdots,
\end{aligned}
$$

where $C_{q}=\left(C_{1, q}, C_{2, q}, \ldots, C_{k, q}\right)^{T}, q=0,1, \ldots$, are constant coefficients (see Ehigie et al. [27]). 
Definition 4. The block method (23) has algebraic order $p \geq$ 1 , provided there exists a constant $C_{p+1} \neq 0$ such that the local truncation error $E_{\mu}$ satisfies $\left\|E_{\mu}\right\|=C_{p+1} h^{p+1}+O\left(h^{p+2}\right)$, where $\|\cdot\|$ is the maximum norm.

Remark 5. (i) The local truncation error constants $\left(C_{p+1}\right)$ of the block method (23) are presented in Table 1. (ii) From the local truncation error constant computation, it follows that the order $(p)$ of the method (23) is $p=k+2$.

\subsection{Stability}

Definition 6. The block method (23) is zero-stable, provided the roots of the first characteristic polynomial have modulus less than or equal to one and those of modulus one are simple (see [2]).

Remark 7. Observe that, from the first characteristic polynomial $\rho_{k}(R)$ of the block method (23) specified by $\rho_{k}(R)=$ $\operatorname{det}\left[\sum_{i=0}^{1} A_{i} R^{i}\right]=0$, we obtain $-R^{k-1}(1+R)=0$. Thus the $\operatorname{roots} R_{j}, j=1,2, \ldots, k$ of $\rho_{k}(R)$ satisfy $\left|R_{j}\right| \leq 1, j=1,2, \ldots, k$ and, for those roots with $\left|R_{j}\right|=1$, the roots are simple.

Definition 8. The block method (23) is consistent if it has order $p>1$ (see [26]).

Remark 9. The block method (23) is consistent as it has order $p>1$ and zero-stable; hence it is convergent since zerostability + consistency $=$ convergence.

Proposition 10. The block method (23) applied to the test equations $y^{\prime}=\lambda y$ and $y^{\prime \prime}=\lambda^{2} y$ yields

$$
\begin{aligned}
Y_{\mu+1} & =M(q ; u) Y_{\mu}, \\
M(q ; u) & =\left(A_{1}-q B_{1}-q^{2} C_{1}\right)^{-1}\left(A_{0}+q B_{0}\right), \\
& q=\lambda h^{2}, u=w h .
\end{aligned}
$$

Proof. We begin by applying (23) to the test equations $y^{\prime}=$ $\lambda y$ and $y^{\prime \prime}=\lambda^{2} y$ which are expressed as $f(x, y)=\lambda y$ and $g(x, y)=\lambda^{2} y$, respectively; letting $q=h \lambda$ and $u=w h$, we obtain a linear equation which is used to solve for $Y_{\mu+1}$ with (23) as a consequence.

Remark 11. The rational function $M(q ; u)$ is called the stability function which determines the stability of the method.

Definition 12. A region of stability is a region in the $q-u$ plane, in which $|M(q ; u)| \leq 1$.

Corollary 13. Method (23) has $M(q ; u)$ specified in Appendix 2 of the supplementary material.

Remark 14. In the $q-u$ plane the $\operatorname{ESDM~(23)~is~stable~for~} q \leq 0$, and $u \in[-2 \pi, 2 \pi]$, since $|M(q ; u)| \leq 1, q \leq 0$.

Remark 15. Figures $1,3,5$, and 7 are plots of the stability region of $M(q ; u)$ for case $k=1,2, \ldots, 4$, respectively. We note from these figures that the stability region of $M(q ; u)$
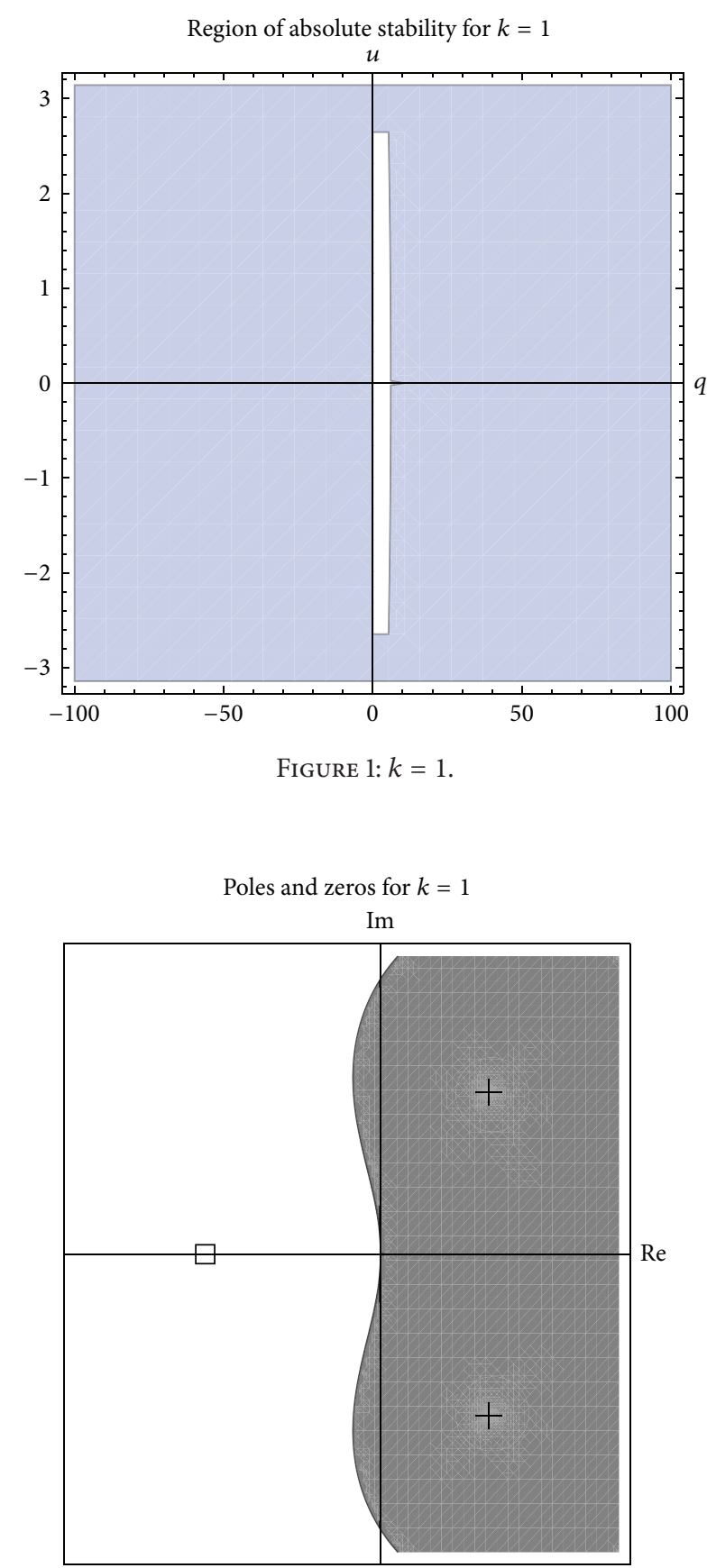

Figure $2: k=1: M(q ; u)$ has zeros $(\square)$ and no poles $(+)$ in $\mathbb{C}^{-}$.

for $k=1,2, \ldots, 4$ includes the entire left side of the complex plane. Figures $2,4,6$, and 8 show the respective zeros and poles of $M(q ; u)$.

3.3. Implementation. The ESDM (10) is implemented in the spirit of Ngwane et al. in [28, 29] to solve (2) without requiring starting values and predictors. For instance, if we let $n=0$ in (10), then $y_{1}, y_{2}, \ldots, y_{k}$ are obtained on the subinterval $\left[x_{0}, x_{k}\right]$, as $y_{0}$ is known from the IVP. If $n=1$, then 


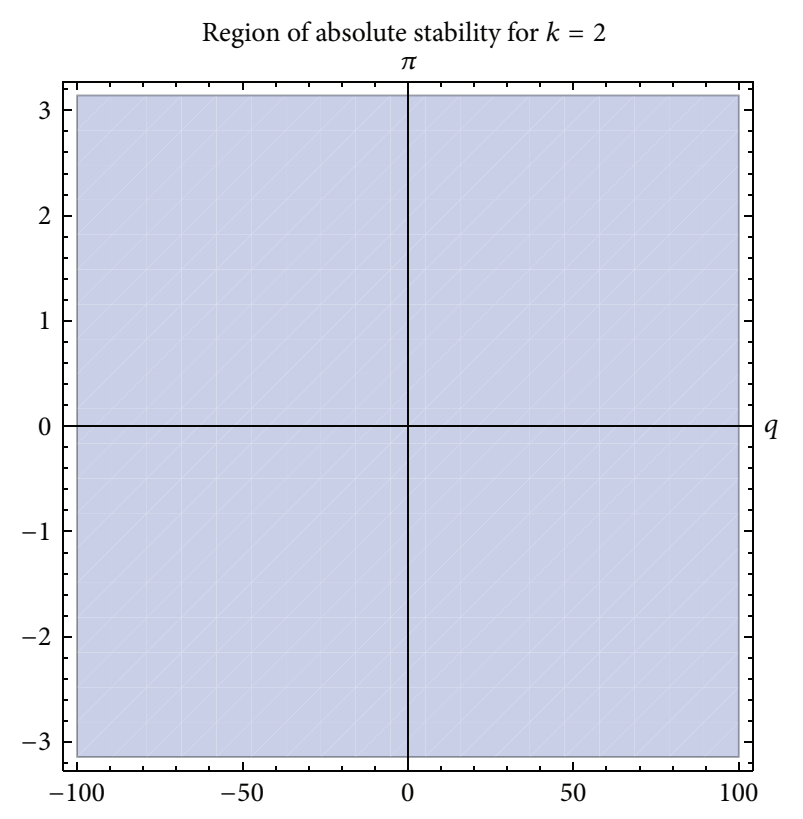

FIGURE 3: $k=2$.

Poles and zeros for $k=2$

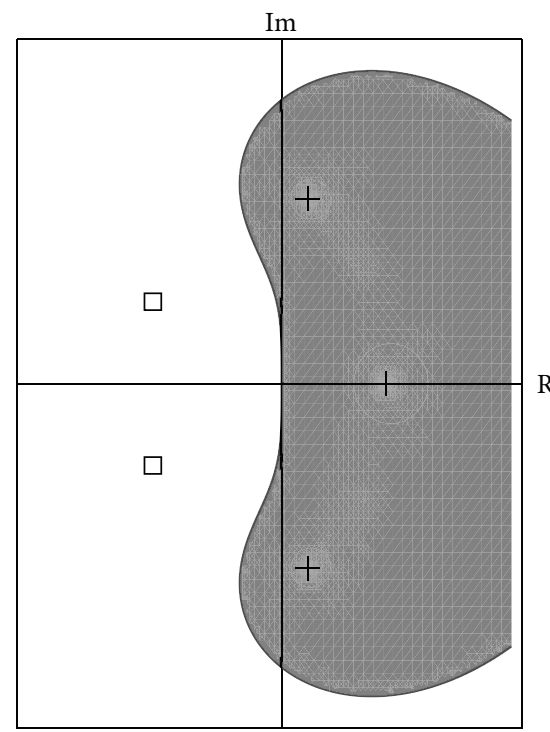

Figure $4: k=2: M(q ; u)$ has zeros $(\square)$ and no poles $(+)$ in $\mathbb{C}^{-}$.

$y_{k+1}, y_{k+2}, \ldots, y_{2 k}$ are obtained on the subinterval $\left[x_{k}, x_{2 k}\right]$, as $y_{k}$ is known from the previous computation and so on, until we reach the final subinterval $\left[x_{N-1}, x_{N}\right]$. Note that, for linear problems, we solve (2) directly using the feature Solve[] in Mathematica 8.0, while for nonlinear problems we use Newton's method enhanced by the feature FindRoot[].

\section{Numerical Illustration}

In this section we consider some standard problems: stiff, oscillatory, linear, and nonlinear systems that appear in

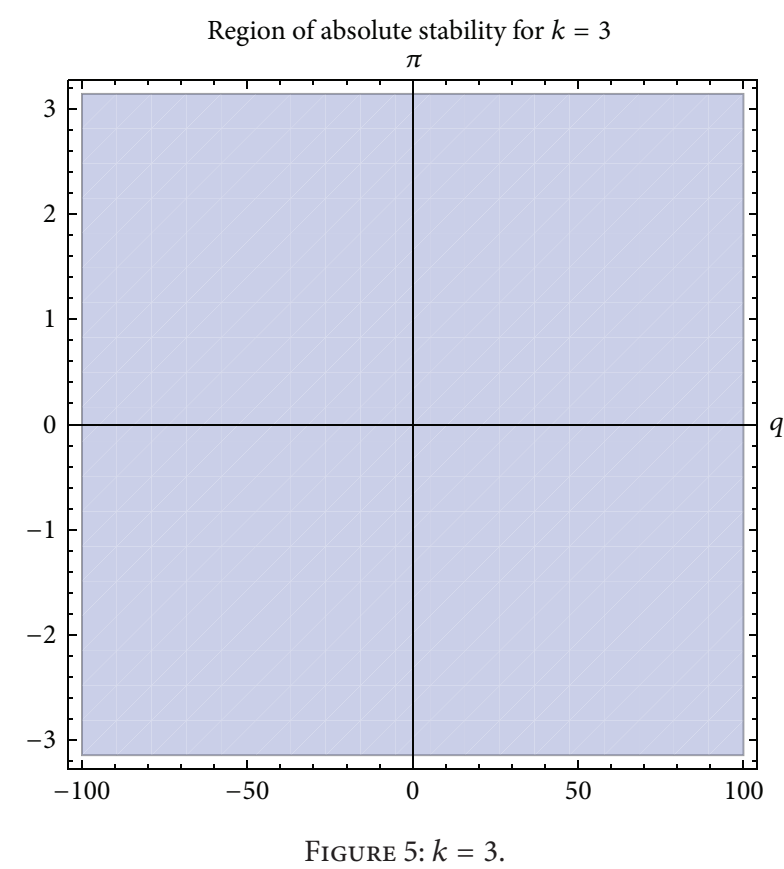

Poles and zeros for $k=3$

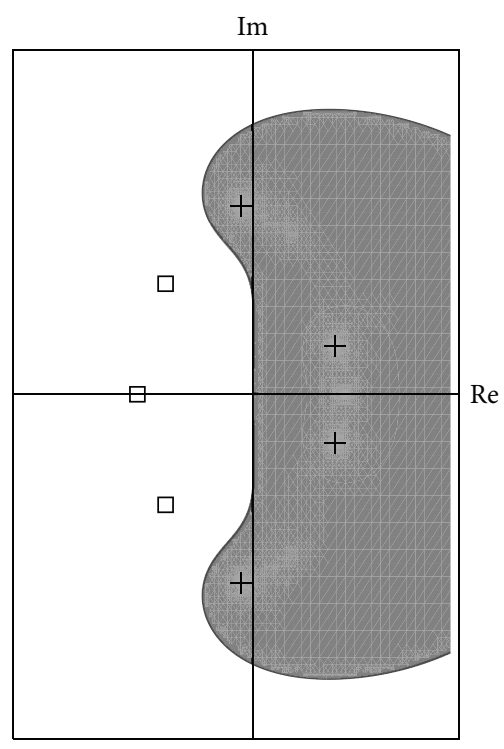

Figure 6: $k=3: M(q ; u)$ has zeros $(\square)$ and poles $(+)$ in $\mathbb{C}^{-}$.

the literature to experimentally illustrate the accuracy and efficiency of the ESDM (10) which is implemented in block form. The ESDM for $k=1,2,3$, and 4 as early stated are denoted by EM1, EM2, EM3, and EM4, respectively. Our numerical examples test this family of methods. We include examples of second-order IVPs and it would be pertinent to mention here that there are methods specifically designed for this type of problems. In this paper, all the numerical experiments are carried out with fixed $h$ and $\omega$, assuming that $\omega$ is known. This allows us to compute the coefficients 


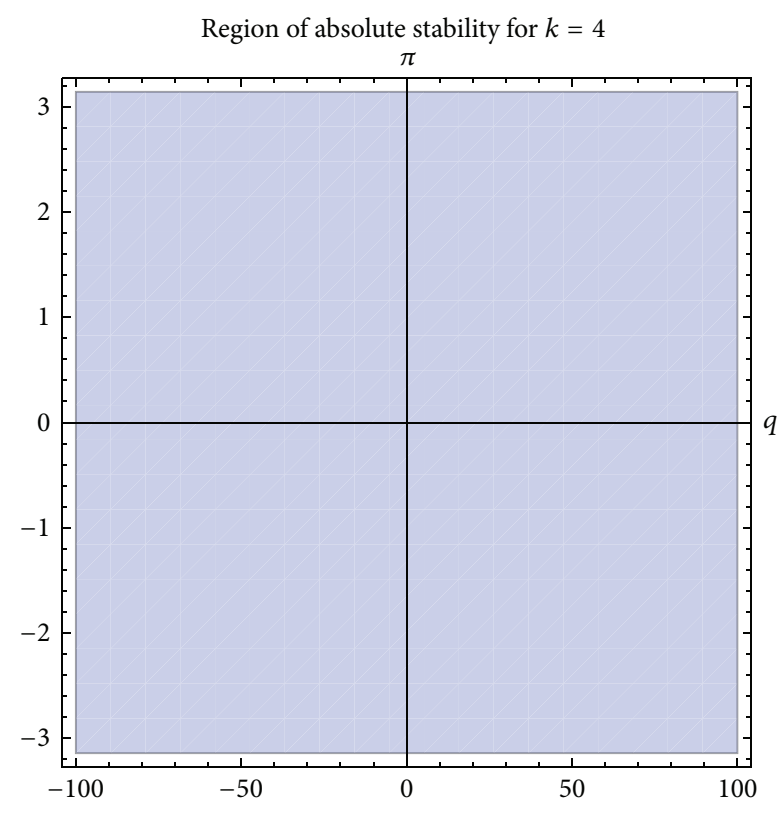

FIGURE $7: k=4$.

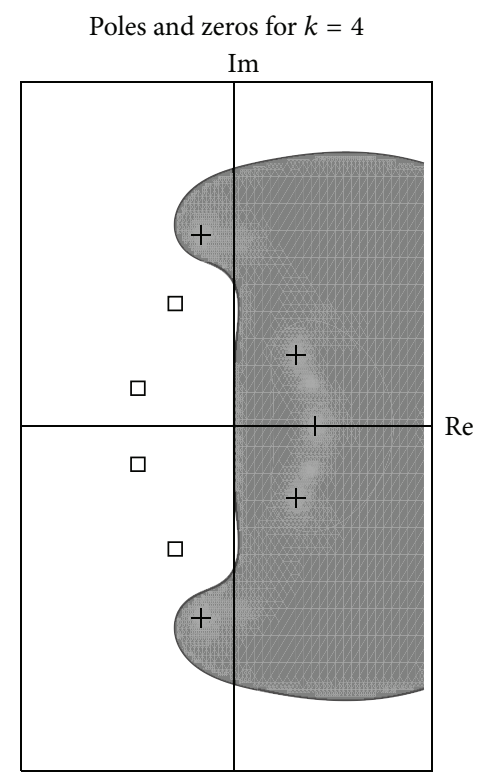

FIGURE $8: k=4: M(q ; u)$ has zeros $(\square)$ and poles $(+)$ in $\mathbb{C}^{-}$.

of the ESDM once for all integration. Some of the methods of orders 4 and 6 in the literature have been compared to EM2 and EM4, respectively. We find the approximate solution on the partition $\pi_{N}$, and we give the errors at the endpoints calculated as Error $=y_{N}-y\left(x_{N}\right)$. We denote the $\operatorname{Max}\left|y_{N}-y\left(x_{N}\right)\right|$ by Err, the number of steps by $N$, and the number of function evaluations by NFEs. We will write an error of the form $\operatorname{Err}=q \times 10^{-r}$ as $q(-r)$.

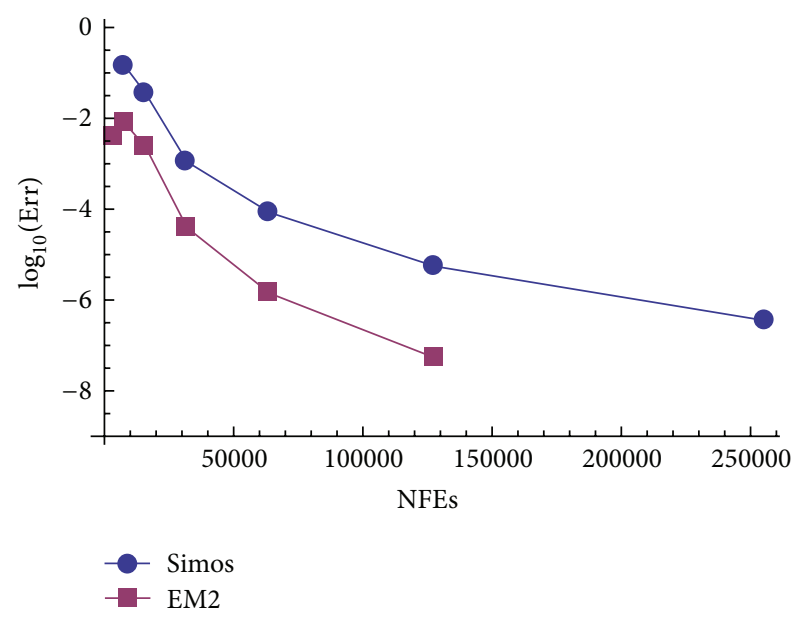

FIgURE 9: Efficiency curves for Example 16.

Example 16. We consider the following inhomogeneous IVP by Simos [25]:

$$
\begin{aligned}
y^{\prime \prime} & =-100 y+99 \sin (x), \\
y(0) & =1, \\
y^{\prime}(0) & =11,
\end{aligned}
$$

$$
x \in[0,1000],
$$

where the analytic solution is given by $y(x)=\cos (10 x)+$ $\sin (10 x)+\sin (x)$.

EM2 is fourth-order and hence comparable to the exponentially fitted method by Simos [25] which is also of fourthorder. PC1 and PC2 denote the predictor-corrector mode for $k=1$ and $k=2$, respectively. The efficiency curves in Figure 9 show the computational efficiency of the two methods (Simos and EM2) by considering the NFEs over $N$ integration steps for each method. Hence for this example, EM2 performs better than Simos. We see from Table 2 that ESDM is efficient for each case.

Example 17. We consider the nonlinear Duffing equation which was also solved by Ixaru and Vanden Berghe [30]:

$$
\begin{aligned}
y^{\prime \prime}+y+y^{3} & =B \cos (\Omega x), \\
y(0) & =C_{0}, \\
y^{\prime}(0) & =0, \\
& x \in[0,300] .
\end{aligned}
$$

The analytic solution is given by $y(x)=C_{1} \cos (\Omega x)+$ $C_{2} \cos (3 \Omega x)+C_{3} \cos (5 \Omega x)+C_{4} \cos (7 \Omega x)$, where $\Omega=1.01$, $B=0.002, C_{0}=0.200426728069, C_{1}=0.200179477536$, $C_{2}=0.246946143 \times 10^{-3}, C_{3}=0.304016 \times 10^{-6}, C_{4}=$ $0.374 \times 10^{-9}$, and $\omega=1.01$.

We compare the errors produced by our EM2 with the fourth-order methods by Ixaru and Vanden Berghe [30]. 
TABLE 2: Results with $\omega=10$, for Example 16.

\begin{tabular}{|c|c|c|c|c|c|c|c|}
\hline \multirow[b]{2}{*}{$N$} & \multicolumn{6}{|c|}{ ESDM } & \multirow{2}{*}{$\begin{array}{c}\text { Simos [25] } \\
\text { Simos } \\
\text { Err }\end{array}$} \\
\hline & $\begin{array}{c}\text { EM1 } \\
\text { Err }\end{array}$ & $\begin{array}{c}\text { EM2 } \\
\text { Err }\end{array}$ & $\begin{array}{c}\text { EM3 } \\
\text { Err }\end{array}$ & $\begin{array}{c}\text { EM4 } \\
\text { Err }\end{array}$ & $\begin{array}{l}\text { PCl } \\
\text { Err }\end{array}$ & $\begin{array}{c}\text { PC2 } \\
\text { Err }\end{array}$ & \\
\hline 1000 & $1.2(-4)$ & $3.9(-3)$ & $2.1(-3)$ & $5.8(-1)$ & 5.11 & 4.24 & $1.4(-1)$ \\
\hline 2000 & $3.7(-2)$ & $7.7(-3)$ & $3.9(-5)$ & $1.7(-4)$ & 2.49 & 8.42 & $3.5(-2)$ \\
\hline 4000 & $4.9(-4)$ & $2.3(-3)$ & $2.3(-4)$ & $8.4(-5)$ & $2.76(-2)$ & 1.835 (1) & $1.1(-3)$ \\
\hline 8000 & $2.3(-5)$ & $3.9(-5)$ & $1.9(-6)$ & $3.4(-7)$ & $2.83(-2)$ & $3.75(1)$ & $8.4(-5)$ \\
\hline 16000 & $6.8(-6)$ & $1.4(-6)$ & $3.4(-8)$ & $2.1(-10)$ & $4.33(-3)$ & $7.47(1)$ & $5.5(-6)$ \\
\hline 32000 & $1.0(-6)$ & $5.3(-8)$ & $2.6(-12)$ & $3.1(-11)$ & $3.79(-4)$ & $1.51(2)$ & $3.5(-7)$ \\
\hline
\end{tabular}

TABLe 3: Results with $\omega=1.01$, for Example 17 .

\begin{tabular}{lccccccc}
\hline & & \multicolumn{2}{c}{ ESDM } & \multicolumn{2}{c}{ Ixaru and Vanden Berghe [30] } \\
$N$ & $\begin{array}{c}\text { EM1 } \\
\text { Err }\end{array}$ & EM2 & $\begin{array}{c}\text { EM3 } \\
\text { Err }\end{array}$ & $\begin{array}{c}\text { EM4 } \\
\text { Err }\end{array}$ & $\begin{array}{c}\text { Simos } \\
\text { Err }\end{array}$ & $\begin{array}{c}\text { Ixaru and } \\
\text { Vanden Err }\end{array}$ \\
\hline 300 & $1.1(-4)$ & $2.8(-4)$ & $4.7(-1)$ & $5.7(-4)$ & $1.7(-3)$ & $1.1(-3)$ \\
600 & $1.8(-5)$ & $2.3(-5)$ & $1.9(-5)$ & $4.5(-6)$ & $1.9(-4)$ & $5.4(-5)$ \\
1200 & $2.7(-6)$ & $1.3(-6)$ & $3.3(-7)$ & $2.9(-7)$ & $1.4(-5)$ & $1.9(-6)$ \\
2000 & $6.2(-7)$ & $1.6(-7)$ & $1.6(-8)$ & $1.2(-8)$ & - & - \\
2400 & $13.7(-7)$ & $5.8(-8)$ & $1.1(-8)$ & $3.9(-9)$ & $8.7(-7)$ & $6.2(-8)$ \\
3000 & $1.9(-7)$ & $1.2(-8)$ & $3.9(-9)$ & $1.1(-9)$ & - & - \\
4800 & $4.8(-8)$ & $7.8(-10)$ & $4.0(-10)$ & $4.1(-11)$ & - & - \\
\hline
\end{tabular}

We see from Table 3 that the results produced by ESDM on Example 17 are very good. In fact, EM2 produces results that are better than Simos' method used in [30], as it produces better error magnitude while using less number of steps and fewer number of function evaluations. This example once more shows us that the ESDM produces good results and in particular EM2 is very competitive to the method used by Ixaru and Vanden Berghe [30].

Example 18 (a nearly sinusoidal problem). We consider the following IVP on the range $0 \leq t \leq 10$ (see the study by Nguyen et al. [16]):

$$
\begin{aligned}
y_{1}^{\prime} & =-2 y_{1}+y_{2}+\sin (t), \\
y_{1}(0) & =2, \\
y_{2}^{\prime} & =-(\beta+2) y_{1}+(\beta+1) y_{2}+\sin (t)-\cos (t), \\
y_{2}(0) & =3 .
\end{aligned}
$$

We choose $\beta=-3$ and $\beta=-1000$ in order to illustrate the phenomenon of stiffness. Given the initial conditions $y_{1}(0)=$ 2 and $y_{2}(0)=3$, the exact solution is $\beta$-independent and is given by

$$
\text { Exact: } \begin{aligned}
y_{1}(t) & =2 \exp (-t)+\sin (t), \\
y_{2}(t) & =2 \exp (-t)+\cos (t) .
\end{aligned}
$$

We choose this example to demonstrate the performance of ESDM on stiff problems. We compute the solutions to Example 18 with $\beta=-3,-1000$. We compare EM4 of order six to the method by Nguyen et al. [16] which is also of order six. For both $\beta=-3$ and $\beta=-1000$, EM4 clearly obtains better absolute errors compared to Nguyen et al. [16]. This efficiency is achieved using fewer number of steps and less number of function evaluations than Nguyen et al. [16].

Example 19. Consider the given two-body problem which was solved by Ozawa [15]:

$$
\begin{gathered}
y_{1}^{\prime \prime}=-\frac{y_{1}}{r^{3}}, \\
y_{2}^{\prime \prime}=-\frac{y_{2}}{r^{3}}, \\
r=\sqrt{y_{1}^{2}+y_{2}^{2}}, \\
y_{1}(0)=1-e, \\
y_{1}^{\prime}(0)=0, \\
y_{2}(0)=0, \\
y_{2}^{\prime}(0)=\sqrt{\frac{1+e}{1-e},} \\
x \in[0,50 \pi], \omega=1,
\end{gathered}
$$

where $e, 0 \leq e<1$, is an eccentricity. The exact solution of this problem is $y_{1}(x)=\cos (k)-e, y_{2}(x)=\sqrt{1-e^{2}} \sin (k)$, where $k$ is the solution of Kepler's equation $k=x+e \sin (k)$.

Table 5 contains the results obtained using the ESDM for $k=1,2,3,4$. These results are compared with the explicit 
singly diagonally implicit Runge-Kutta (ESDIRK) and the functionally fitted ESDIRK (FESDIRK) methods given in Ozawa [15]. In terms of accuracy, Table 5 clearly shows that ESDM performs better than those in Ozawa [15].

4.1. A Predictor-Corrector Mode Implementation of ESDM. We can implement our ESDM in a predictor-corrector (PC) mode. The predictor for $k=1,2,3,4$ is given below while the corrector for each case is given by the main method (10). PC1 and PC2 denote the PC mode for $k=1$ and 2, respectively:

$$
\begin{aligned}
& k=1: \\
& y_{n+1}=y_{n}+\frac{h \sin u}{u} f_{n}+\frac{2 h^{2} \sin (u / 2)^{2}}{u^{2}} g_{n}, \\
& k=2: \\
& y_{n+2}=y_{n+1}-\frac{h \csc (u / 2)^{2}(-u+\sin u)}{2 u} f_{n}
\end{aligned}
$$

$$
\begin{aligned}
& \bar{\beta}_{0}=-\frac{\left(\csc (u / 2)^{2}\left(2-3 u^{2}-2 \cos u+\left(-2+u^{2}\right) \cos u+2 \cos (2 u)+4 u \sin u\right)\right)}{(8 u(u \cos u-\sin u))} ; \\
& \bar{\beta}_{1}=\frac{\left(\csc (u / 2)^{2}\left(2-5 u^{2}-2 \cos u+\left(-2+u^{2}\right) \cos (2 u)+2 \cos (3 u)+4 u \sin u+2 u \sin (2 u)\right)\right)}{(8 u(u \cos u-\sin u))} ; \\
& \bar{\beta}_{2}=-\frac{\left(\csc (u / 2)^{2}\left(\left(2-5 u^{2}\right) \cos u+\left(-2+3 u^{2}\right) \cos (2 u)+2(-\cos (2 u)+\cos (3 u)+2 u \sin u)\right)\right)}{(8 u(u \cos u-\sin u))} ; \\
& \bar{\gamma}_{n+2}=\frac{\left(\csc (u / 2)^{2}(-2+6 \cos u-6 \cos (2 u)+2 \cos (3 u)-6 u \sin u+3 u \sin (2 u))\right)}{(8 u(u \cos u-\sin u))} .
\end{aligned}
$$

4.2. Estimating the Frequency. Though we are mainly interested in problems where $\omega$ is taken as the exact frequency of the analytical solution and $\omega$ is known in advance, it is important to note that the exact frequency may be unknown for some problems. A preliminary testing indicates that a good estimate of the frequency can be obtained by demanding that the LTE in Theorem 3 equals zero and solving for the frequency. That is, solve for $\omega$ given that $C_{k+3} h^{k+3}\left(\omega^{2} y^{(k+1)}\left(x_{n}\right)+y^{(k+3)}\left(x_{n}\right)\right)=0$, where $y^{(j)}, j=$ $k+1, k+3$, denotes derivatives. We used this procedure to estimate $\omega$ for the problem given in Example 16 and obtained $\omega \approx \pm 9.999996$, which approximately gives the known frequency $\omega=10$. Hence, this procedure is interesting and will be seriously considered in our future research.

If a problem has multiple frequencies, then $\omega$ is approximatively calculated so that it is an indicative frequency (see Nguyen et al. [16]). We note that estimating the frequency when it is unknown as well as finding the frequency for problems for which the frequency varies over time is very challenging. This challenge and the choice of the frequency in trigonometrically fitted methods have grown in interest. Existing references on how to estimate the frequency and the choice of the frequency include Vanden Berghe et al. [31] and Ramos and Vigo-Aguiar [32].

\section{Conclusion}

In this paper, we have proposed a family of Enright methods using trigonometric bases for solving stiff and oscillatory IVPs. The ESDM is zero-stable and produced good results on stiff IVPs. This method has the advantages of being selfstarting and having good accuracy properties. ESDM has order $(k+2)$ similar to that in Enright [22]. We have presented representative numerical examples that are linear, nonlinear, stiff, and highly oscillatory. The need that the frequency be known in advance might be a shortcoming, yet these examples show that the ESDM is not only promising but more accurate and efficient than those in Nguyen et al. [16], Simos [25], Ixaru and Vanden Berghe [30], and Ozawa [15]. Details of the numerical results are displayed in Tables 2, 3, 4, and 5, and the efficiency curves are presented in Figures 9, 10, 11, 12 , and 13 . Our future research will incorporate a technique for accurately estimating the frequency as suggested in 


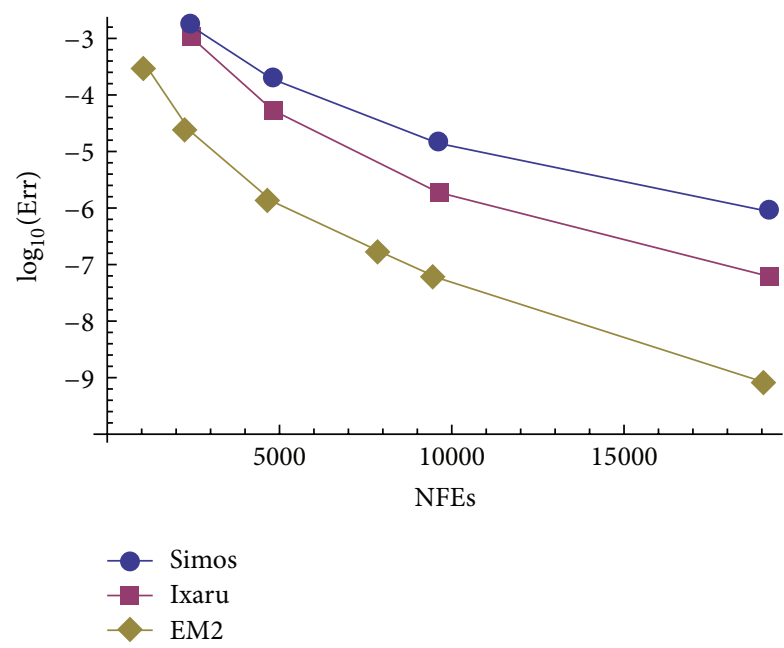

FIGURE 10: Efficiency curves for Example 17.

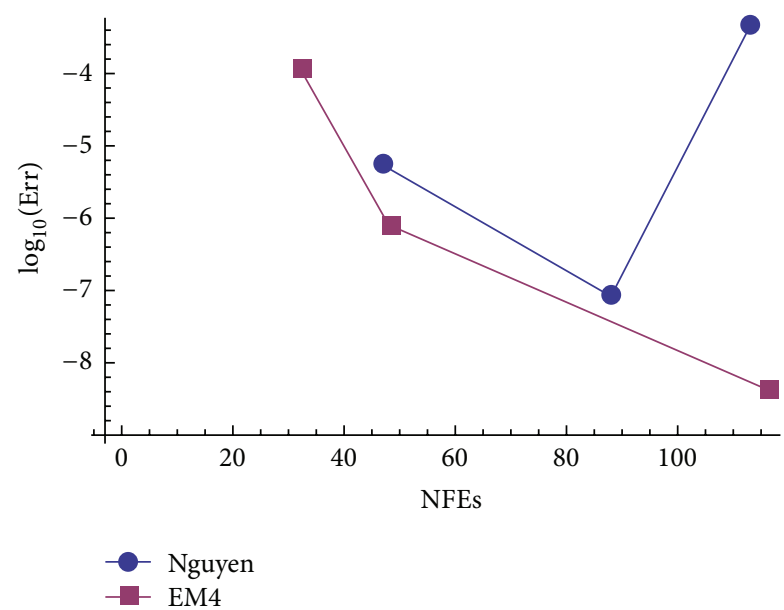

FIgURE 11: Efficiency curves for Example 18 with $\beta=-3$.

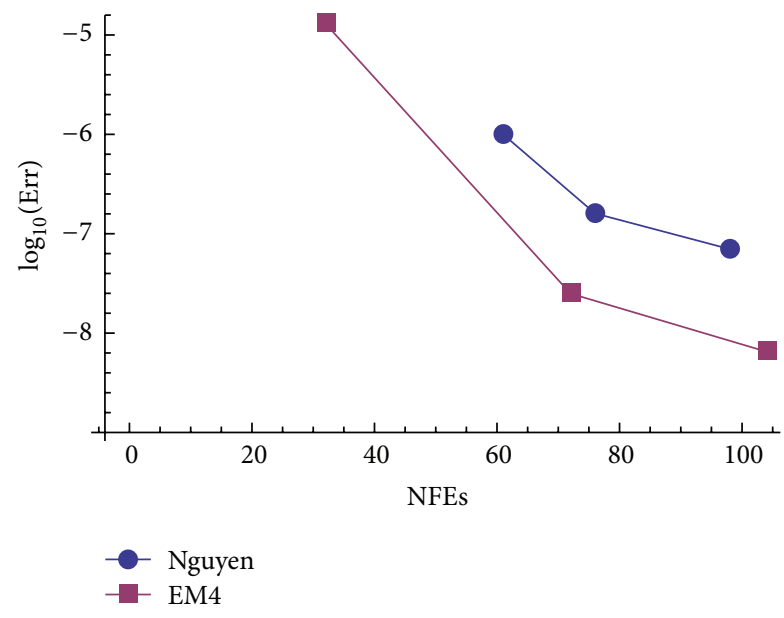

FIGURE 12: Efficiency curves for Example 18 with $\beta=-1000$.
TABLE 4: Results with $\omega=1$, for Example 18.

\begin{tabular}{|c|c|c|c|c|c|}
\hline & \multicolumn{4}{|c|}{ ESDM } & \multirow[t]{2}{*}{ Nguyen et al. [16] } \\
\hline \multicolumn{5}{|c|}{ For $\beta=-3$ we have } & \\
\hline & EM1 & EM2 & EM3 & EM4 & Nguyen \\
\hline$N$ & 6 & 6 & 6 & 6 & - \\
\hline Err & $6.6(-5)$ & $3.8(-5)$ & $7.1(-5)$ & $1.2(-4)$ & - \\
\hline$N$ & 10 & 10 & 10 & 10 & 10 \\
\hline Err & $1.9(-5)$ & $1.3(-6)$ & $4.1(-6)$ & $8.1(-7)$ & $5.4(-6)$ \\
\hline$N$ & 27 & 27 & 27 & 27 & 19 \\
\hline Err & $1.2(-6)$ & $8.2(-8)$ & $3.1(-8)$ & $4.3(-9)$ & $8.3(-8)$ \\
\hline$N$ & 32 & 32 & 32 & 32 & 23 \\
\hline Err & $7.1(-7)$ & $6.3(-8)$ & $1.3(-8)$ & $1.9(-9)$ & $4.5(-4)$ \\
\hline \multicolumn{6}{|c|}{ For $\beta=-1000$ we have } \\
\hline & EM1 & EM2 & EM3 & EM4 & Nguyen \\
\hline$N$ & 6 & 6 & 6 & 6 & 13 \\
\hline Err & $6.6(-5)$ & $3.8(-5)$ & $7.1(-5)$ & $1.3(-5)$ & $1.0(-6)$ \\
\hline$N$ & 16 & 16 & 16 & 16 & 16 \\
\hline Err & $5.3(-6)$ & $6.2(-7)$ & $1.8(-7)$ & $2.5(-8)$ & $1.6(-7)$ \\
\hline$N$ & 20 & 20 & 24 & 24 & 21 \\
\hline Err & $2.8(-6)$ & $3.2(-7)$ & $5.3(-8)$ & $6.5(-9)$ & $7.0(-8)$ \\
\hline
\end{tabular}

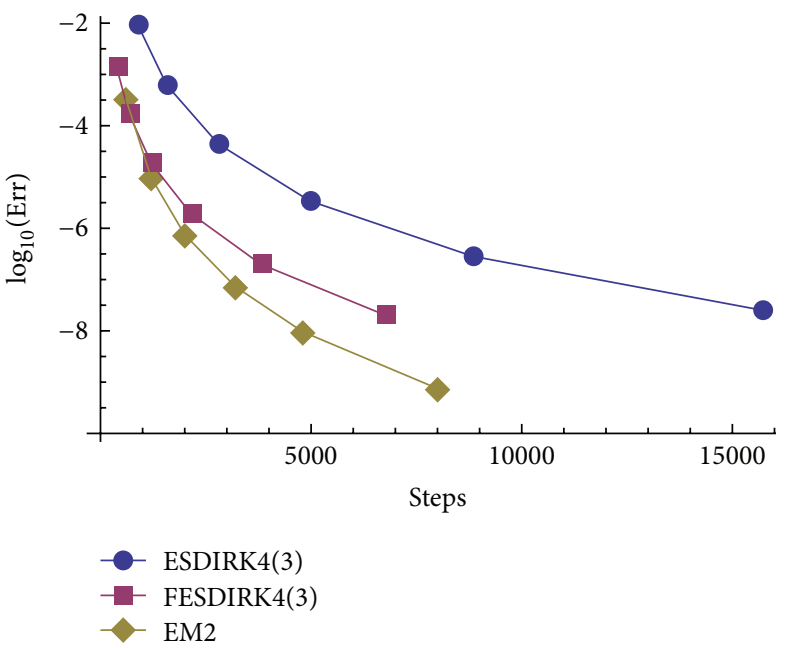

FIgURE 13: Efficiency curves for Example 19.

Section 4.2 as well as implementing the method in a variable step mode.

\section{Conflict of Interests}

The authors declare that there is no conflict of interests regarding the publication of this paper. 
TABLE 5: Results with $\omega=1, e=0.005$, for Example 19.

\begin{tabular}{|c|c|c|c|c|c|c|c|}
\hline & \multicolumn{5}{|c|}{ ESDM } & \multirow{2}{*}{$\begin{array}{c}\text { FESDIRK } \\
\text { FESDIRK4 (3) }\end{array}$} & \multirow{2}{*}{$\begin{array}{c}\text { ESDIRK } \\
\text { ESDIRK4 (3) }\end{array}$} \\
\hline & EM1 & EM2 & EM3 & EM4 & EM5 & & \\
\hline$N$ & 300 & 600 & 600 & 600 & 1200 & 381 & 884 \\
\hline Err & $2.4(-3)$ & $3.0(-4)$ & $1.2(-4)$ & $2.6(-1)$ & $1.2(-7)$ & $1.4(-3)$ & $9.4(-3)$ \\
\hline$N$ & 600 & 1200 & 1200 & 1200 & 2000 & 680 & 1573 \\
\hline Err & $1.8(-3)$ & $9.8(-6)$ & $4.5(-6)$ & $4.6(-7)$ & $2.4(-9)$ & $1.7(-4)$ & $6.2(-4)$ \\
\hline$N$ & 1200 & 2000 & 2000 & 2000 & 2400 & 1207 & 2796 \\
\hline Err & $2.8(-4)$ & $7.5(-7)$ & $4.0(-7)$ & $1.3(-8)$ & $1.1(-9)$ & $1.8(-5)$ & $4.4(-5)$ \\
\hline$N$ & 2000 & 3200 & 3200 & 3200 & 3200 & 2144 & 4970 \\
\hline Err & $6.2(-5)$ & $7.3(-8)$ & $3.9(-8)$ & $4.9(-10)$ & $1.6(-10)$ & $1.9(-6)$ & $3.4(-6)$ \\
\hline$N$ & 4000 & 4800 & 4800 & 4800 & 4800 & 3806 & 8833 \\
\hline Err & $7.8(-6)$ & $9.6(-9)$ & $5.2(-9)$ & $2.9(-11)$ & $9.2(-12)$ & $1.9(-7)$ & $2.8(-7)$ \\
\hline$N$ & 8000 & 8000 & 8000 & 8000 & 8000 & 6762 & 15706 \\
\hline Err & $9.7(-7)$ & $7.5(-10)$ & $4.0(-10)$ & $8.8(-13)$ & $3.6(-13)$ & $2.0(-8)$ & $2.5(-8)$ \\
\hline
\end{tabular}

\section{Acknowledgment}

This research was funded by the RISE Grant no. 176601332896 of the University of South Carolina, Columbia, SC, USA.

\section{References}

[1] S. O. Fatunla, "Numerical integrators for stiff and highly oscillatory differential equations," Mathematics of Computation, vol. 34, no. 150, pp. 373-390, 1980.

[2] J. D. Lambert, Numerical Methods for Ordinary Differential Systems, John Wiley \& Sons, New York, NY, USA, 1991.

[3] J. D. Lambert, Computational Methods in Ordinary Differential Equations, John Wiley \& Sons, New York, NY, USA, 1973.

[4] C. W. Gear, "Hybrid methods for initial value problems in ordinary differential equations," SIAM Journal on Numerical Analysis, vol. 2, pp. 69-86, 1965.

[5] C. W. Gear, "The automatic integration of stiff ODEs," in Proceedings of the IFIP Congress, vol. 1, pp. 187-194, NorthHolland, Amsterdam, 1968.

[6] E. Hairer, "A one-step method of order 10 for $y^{\prime \prime}=f(x, y)$," IMA Journal of Numerical Analysis, vol. 2, no. 1, pp. 83-94, 1982.

[7] E. Hairer and G. Wanner, Solving Ordinary Differential Equations II, Springer, New York, NY, USA, 1996.

[8] G. G. Dahlquist, "Numerical integration of ordinary differential equations," Mathematica Scandinavica, vol. 4, pp. 69-86, 1956.

[9] C. W. Gear, "Algorithm 407: DIFSUB for solution of ODEs," Communications of the ACM, vol. 14, pp. 185-190, 1971.

[10] W. B. Gragg and H. J. Stetter, "Generalized multistep predictorcorrector methods," Journal of the Association for Computing Machinery, vol. 11, pp. 188-209, 1964.

[11] J. C. Butcher, "A modified multistep method for the numerical integration of ordinary differential equations," Journal of the Association for Computing Machinery, vol. 12, pp. 124-135, 1965.

[12] J. C. Butcher, Numerical Methods for Ordinary Differential Equations, Wiley, Chichester, UK, 2008.

[13] L. Brugnano and D. Trigiante, Solving ODEs by Multistep Initial and Boundary Value Methods, Gordon \& Breach, Amsterdam, The Netherlands, 1998.

[14] L. Brugnano and D. Trigiante, "Block implicit methods for ODEs," in Recent Trends in Numerical Analysis, D. Trigiante,
Ed., pp. 81-105, Nova Science Publishers, New York, NY, USA, 2001.

[15] K. Ozawa, "A functionally fitted three-stage explicit singly diagonally implicit Runge-Kutta method," Japan Journal of Industrial and Applied Mathematics, vol. 22, no. 3, pp. 403-427, 2005.

[16] H. S. Nguyen, R. B. Sidje, and N. H. Cong, "Analysis of trigonometric implicit Runge-Kutta methods," Journal of Computational and Applied Mathematics, vol. 198, no. 1, pp. 187-207, 2007.

[17] G. V. Berghe and M. van Daele, "Exponentially-fitted Obrechkoff methods for second-order differential equations," Applied Numerical Mathematics, vol. 59, no. 3-4, pp. 815-829, 2009.

[18] J. Vigo-Aguiar and H. Ramos, "A family of A-stable Runge-Kutta collocation methods of higher order for initial-value problems," IMA Journal of Numerical Analysis, vol. 27, no. 4, pp. 798-817, 2007.

[19] M. Calvo, J. M. Franco, J. I. Montijano, and L. Rández, "Sixthorder symmetric and symplectic exponentially fitted RungeKutta methods of the Gauss type," Journal of Computational and Applied Mathematics, vol. 223, no. 1, pp. 387-398, 2009.

[20] N. Obrechkoff, Neue Quadraturformeln, vol. 14 of Abhandlungen der Preussischen Akademie der Wissenschaften. Math.Naturw, Akademie der Wissenschaften, 1940.

[21] J. R. Cash, "Second derivative extended backward differentiation formulas for the numerical integration of stiff systems," SIAM Journal on Numerical Analysis, vol. 18, no. 1, pp. 21-36, 1981.

[22] W. H. Enright, "Second derivative multistep methods for stiff ordinary differential equations," SIAM Journal on Numerical Analysis, vol. 11, pp. 321-331, 1974.

[23] H. S. Nguyen, R. B. Sidje, and N. H. Cong, "Analysis of trigonometric implicit Runge-Kutta methods," Journal of Computational and Applied Mathematics, vol. 198, no. 1, pp. 187-207, 2007.

[24] S. N. Jator, S. Swindle, and R. French, "Trigonometrically fitted block Numerov type method for $y^{\prime \prime}=f\left(x, y, y^{\prime}\right)$," Numerical Algorithms, vol. 62, no. 1, pp. 13-26, 2013.

[25] T. E. Simos, "An exponentially-fitted Runge-Kutta method for the numerical integration of initial-value problems with 
periodic or oscillating solutions," Computer Physics Communications, vol. 115, no. 1, pp. 1-8, 1998.

[26] S. O. Fatunla, "Block methods for second order IVPs," International Journal of Computer Mathematics, vol. 41, pp. 55-63, 1991.

[27] J. O. Ehigie, S. N. Jator, A. B. Sofoluwe, and S. A. Okunuga, "Boundary value technique for initial value problems with continuous second derivative multistep method of Enright," Computational \& Applied Mathematics, vol. 33, no. 1, pp. 81-93, 2014.

[28] F. F. Ngwane and S. N. Jator, "Block hybrid-second derivative method for stiff systems," International Journal of Pure and Applied Mathematics, vol. 80, no. 4, pp. 543-559, 2012.

[29] F. F. Ngwane and S. N. Jator, "Block hybrid method using trigonometric basis for initial value problems with oscillating solutions," Numerical Algorithms, vol. 63, no. 4, pp. 713-725, 2013.

[30] L. G. Ixaru and G. Vanden Berghe, Exponential Fitting, Kluwer Academic Publishers, Dordrecht, The Netherlands, 2004.

[31] G. Vanden Berghe, L. G. Ixaru, and H. De Meyer, "Frequency determination and step-length control for exponentially-fitted Runge-Kutta methods," Journal of Computational and Applied Mathematics, vol. 132, no. 1, pp. 95-105, 2001.

[32] H. Ramos and J. Vigo-Aguiar, "On the frequency choice in trigonometrically fitted methods," Applied Mathematics Letters, vol. 23, no. 11, pp. 1378-1381, 2010. 


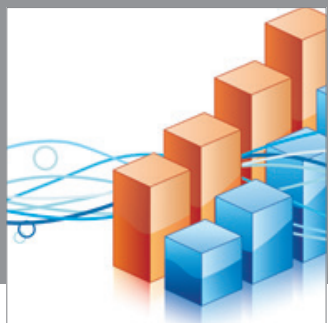

Advances in

Operations Research

mansans

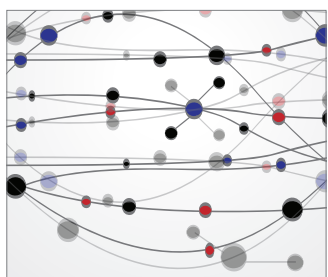

The Scientific World Journal
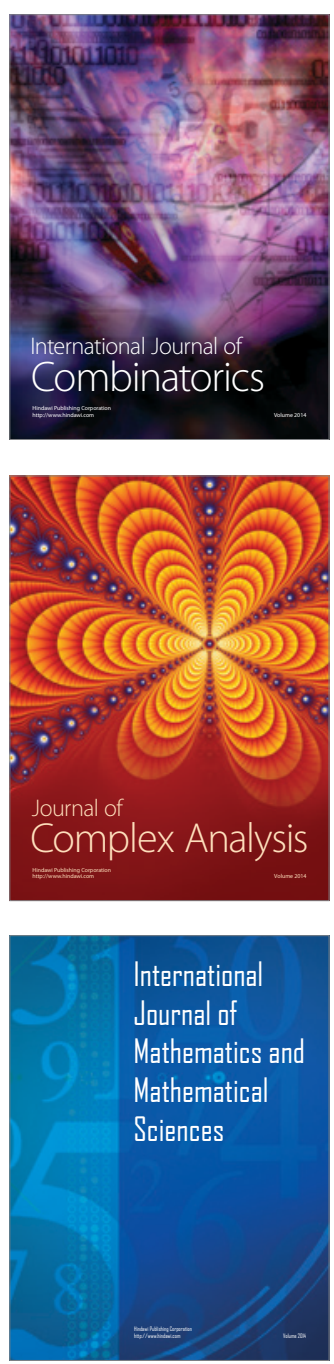
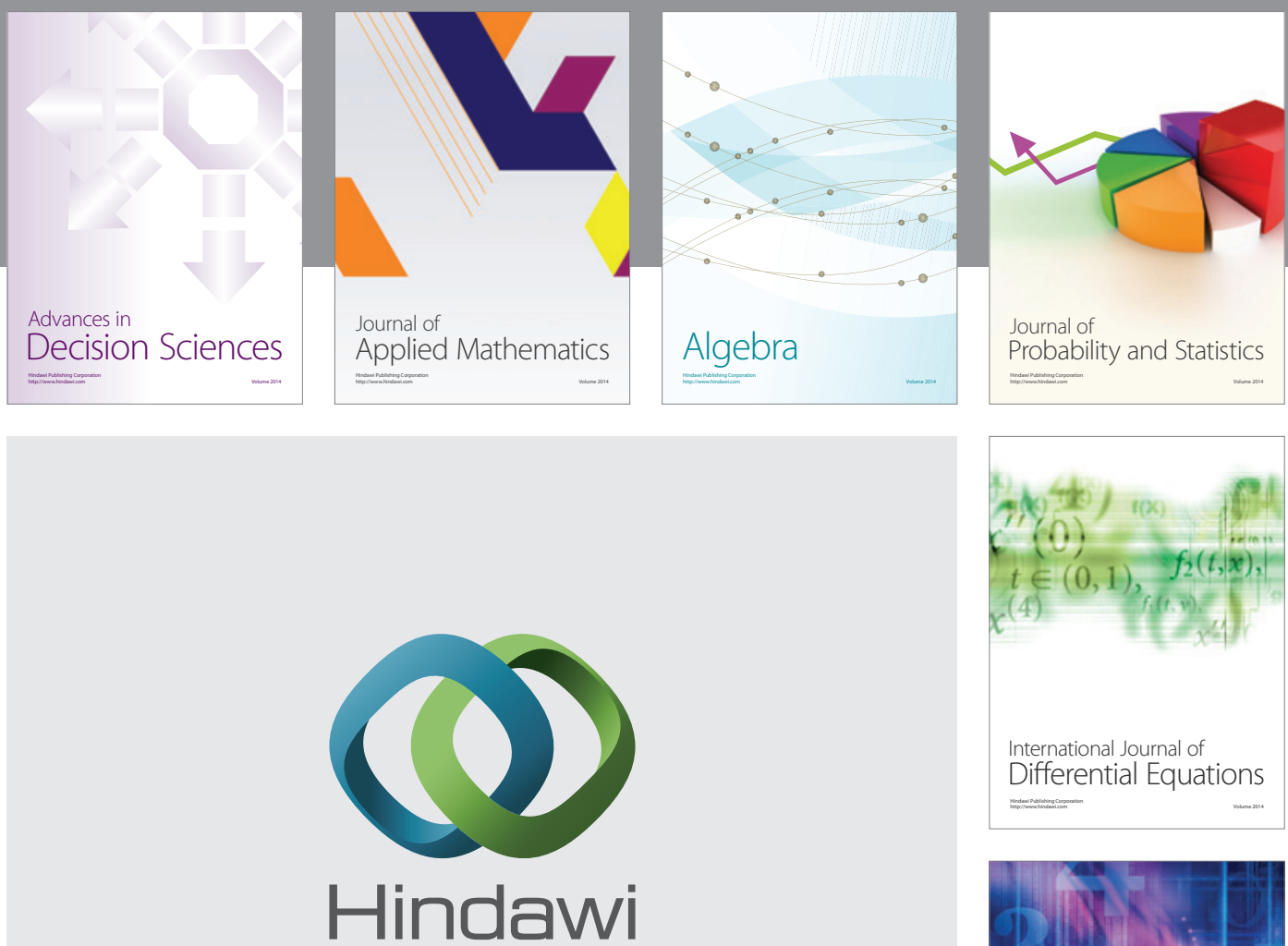

Submit your manuscripts at http://www.hindawi.com
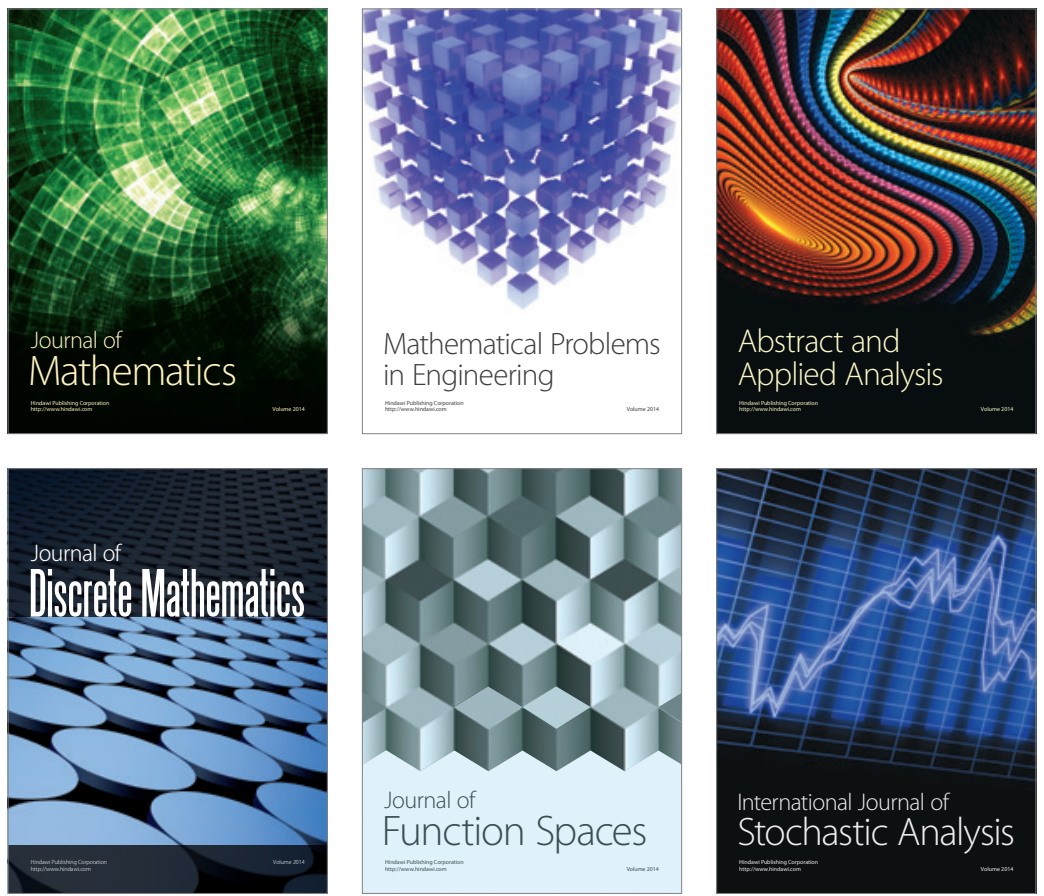

Journal of

Function Spaces

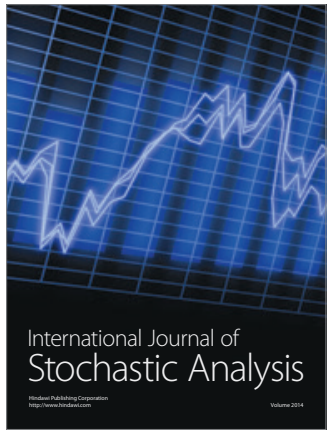

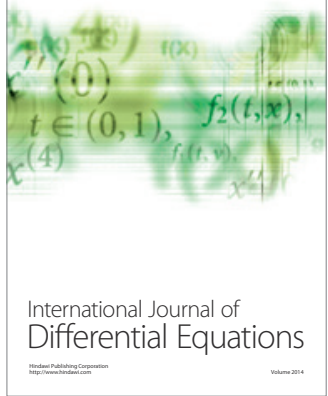
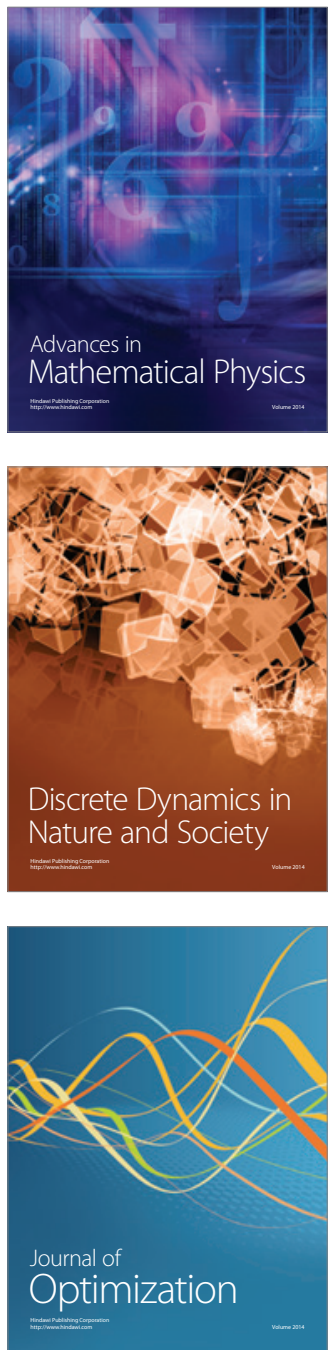